\title{
Salmon Redd Identification Using Environmental DNA (eDNA)
}

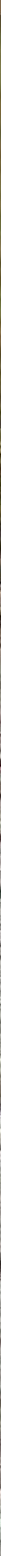

3. Open-File Report 2016-1091

c.

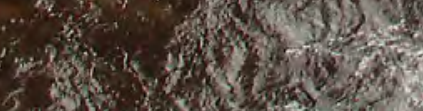

U.S. Department of the Interior

U.S. Geological Survey 
Cover: Photograph of salmon spawning area in the Bull Run River in the Sandy River Basin, Oregon.

Photograph by Burke Strobel, Portland Water Bureau, October 19, 2009. 


\section{Salmon Redd Identification Using Environmental DNA (eDNA)}

By David S. Pilliod and Matthew B. Laramie

Prepared in cooperation with Portland Water Bureau

Open-File Report 2016-1091

U.S. Department of the Interior U.S. Geological Survey 


\section{U.S. Department of the Interior \\ SALLY JEWELL, Secretary}

\section{U.S. Geological Survey \\ Suzette M. Kimball, Director}

U.S. Geological Survey, Reston, Virginia: 2016

For more information on the USGS—-the Federal source for science about the Earth,

its natural and living resources, natural hazards, and the environment-visit

http://www.usgs.gov/ or call 1-888-ASK-USGS (1-888-275-8747).

For an overview of USGS information products, including maps, imagery, and publications, visit http://store.usgs.gov.

Any use of trade, firm, or product names is for descriptive purposes only and does not imply endorsement by the U.S. Government.

Although this information product, for the most part, is in the public domain, it also may contain copyrighted materials as noted in the text. Permission to reproduce copyrighted items must be secured from the copyright owner.

Suggested citation:

Pilliod, D.S., and Laramie, M.B., 2016, Salmon redd identification using environmental DNA (eDNA): U.S. Geological Survey Open-File Report 2016-1091, 25 p., http://dx.doi.org/10.3133/ofr20161091.

ISSN 2331-1258 (online) 


\section{Contents}

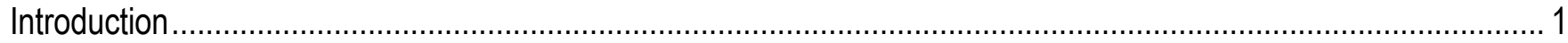

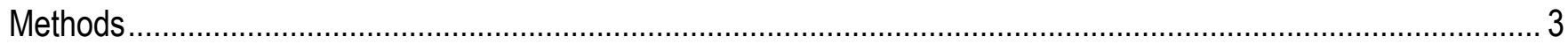

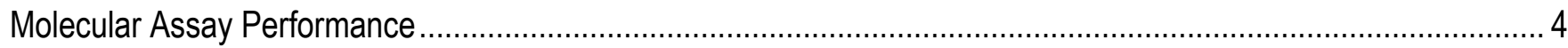

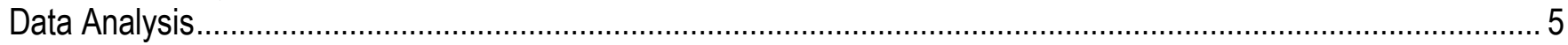

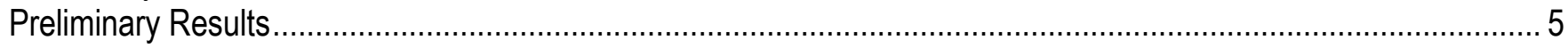

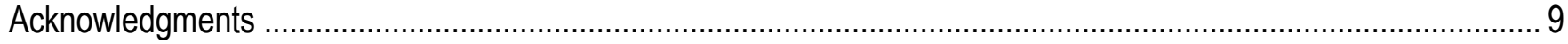

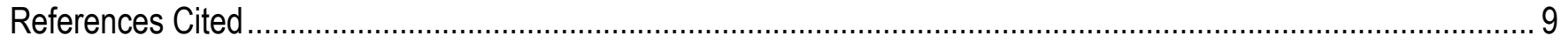

Appendix 1. Environmental DNA (eDNA) Concentrations for Coho Salmon (O. kisutch) and Chinook salmon

(O. tshawytscha) from All Sites Sampled in the Sandy River Basin, northwestern Oregon, fall and winter 2013 ..... 10

Appendix 2. Difference $(\Delta)$ Between Mean Environmental DNA (eDNA) Concentrations near the Substrate and in the Water Column (for O. kisutch and O. tshawytscha) at Each Site in the Sandy River Basin, northwestern Oregon, fall and winter 2013

\section{Figures}

Figure 1. Map showing Sandy River Basin in northwestern Oregon .

Figure 2. Graphs showing (a) Coho salmon (O. kisutch) DNA Standards and (b) Chinook salmon (O. tshawytscha) DNA Standards

Figure 3. Boxplots showing (a) distribution of Coho salmon (O. kistuch) eDNA concentrations at each sample site type, and (b) distribution of Chinook salmon (O. tshawytscha) eDNA concentrations at each sample site type.

Figure 4. Graphs showing (a) comparisons of mean Coho salmon ( 0 . kisutch) eDNA concentrations between all site types, (b) comparisons of mean Chinook salmon( 0 . tshawytscha) eDNA concentrations between all site types

\section{Tables}

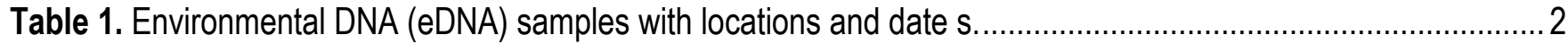

Table 2. Specifications of molecular assays for Coho salmon (O. kisutch) and Chinook salmon (O. tshawytscha)... 3

Table 3. Analysis of Variance (ANOVA) table for differences in Coho salmon (O. kisutch) eDNA among sample types.

Table 4. Tukey multiple comparisons of means with 95 percent family-wise confidence level for Coho salmon (O. kisutch) eDNA among sample types.

Table 5. Analysis of Variance (ANOVA) table for differences in Chinook salmon (O. tshawytscha) environmental DNA (eDNA) among sample types.

Table 6. Tukey multiple comparisons of means with 95 percent family-wise confidence level for Chinook salmon (O. tshawytscha) environmental DNA (eDNA) among sample types. 


\section{Conversion Factors}

Inch/Pound to International System of Units

\begin{tabular}{|c|c|c|}
\hline Multiply & By & To obtain \\
\hline foot (ft) & 0.3048 & meter $(\mathrm{m})$ \\
\hline mile (mi) & 1.609 & kilometer $(\mathrm{km})$ \\
\hline \multicolumn{3}{|c|}{ International System of Units to Inch/Pound } \\
\hline Multiply & By & To obtain \\
\hline kilometer (km) & 0.6214 & mile (mi) \\
\hline milliliter $(\mathrm{mL})$ & 0.033814 & fluid ounce (fl oz) \\
\hline
\end{tabular}

Temperature in degrees Celsius $\left({ }^{\circ} \mathrm{C}\right)$ may be converted to degrees Fahrenheit $\left({ }^{\circ} \mathrm{F}\right)$ as ${ }^{\circ} \mathrm{F}=\left(1.8 \times{ }^{\circ} \mathrm{C}\right)+32$.

\section{Datum}

Horizontal coordinate information is referenced to the North American Datum of 1983 (NAD 83). 


\title{
Salmon Redd Identification Using Environmental DNA (eDNA)
}

\author{
By David S. Pilliod and Matthew B. Laramie
}

\section{Introduction}

The purpose of this project was to develop a technique to use environmental DNA (eDNA) to distinguish between redds made by Chinook salmon (Oncorhynchus tshawytscha) and redds made by Coho salmon (O. kisutch) and to distinguish utilized redds from test/abandoned redds or scours that have the appearance of redds. The project had two phases:

Phase 1. Develop, test, and optimize a molecular assay for detecting and identifying Coho salmon DNA and differentiating it from Chinook salmon DNA.

Phase 2. Demonstrate the efficacy of the technique.

a. Collect and preserve water samples from the interstitial spaces of 10 known redds (as identified by expert observers) of each species and 10 gravel patches that do not include a redd of either species.

b. Collect control samples from the water column adjacent to each redd to establish background eDNA levels.

c. Analyze the samples using the developed molecular assays for Coho salmon (phase I) and Chinook salmon (Laramie and others, 2015).

d. Evaluate whether samples collected from Chinook and Coho redds have significantly higher levels of eDNA of the respective species than background levels (that is, from gravel, water column).

e. Evaluate whether samples collected from the interstitial spaces of gravel patches that are not redds are similar to background eDNA levels.

The Sandy River is a large tributary of the Columbia River (fig. 1). The Sandy River meets the Columbia River approximately $23 \mathrm{~km}$ upstream of Portland, Oregon. The Sandy River Basin provides overlapping spawning habitat for both Chinook and Coho salmon.

Samples provided by Portland Water Bureau for analysis were collected from the Bull Run River, Sixes Creek, Still Creek, Arrah Wanna Side Channel, and Side Channel 18 (table 1). 


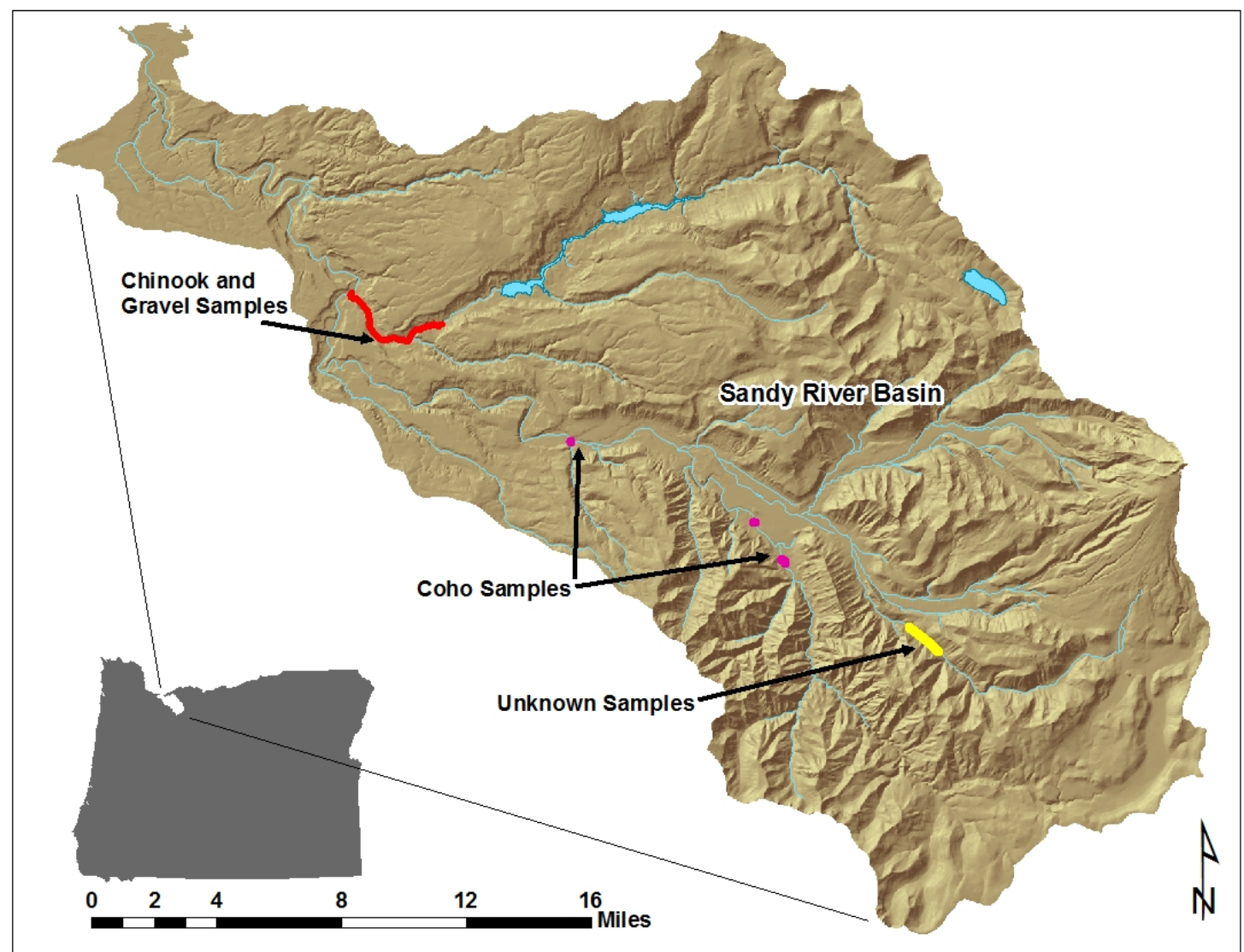

Figure 1. Map showing Sandy River Basin in northwestern Oregon. (Map provided by Burke Strobel, Portland Water Bureau on 23 February 2015.) Approximate location of study area: 45024'09.94"N, 122001'29.40"W.

Table 1. Environmental DNA (eDNA) samples with locations and date $\mathrm{s}$.

[Number of samples: Each site consisted of seven samples, including three redd/gravel replicates, three water column replicates, and one distilled water control]

\begin{tabular}{lccc}
\hline \multicolumn{1}{c}{ Sample location } & $\begin{array}{c}\text { Date sample } \\
\text { collected }\end{array}$ & $\begin{array}{c}\text { Number of } \\
\text { sites }\end{array}$ & $\begin{array}{c}\text { Number of } \\
\text { samples }\end{array}$ \\
\hline Bull Run River & $11 / 14 / 2013$ & 10 & 70 \\
Bull Run River & $11 / 26 / 2013$ & 10 & 70 \\
Sixes Creek & $12 / 18 / 2013$ & 4 & 28 \\
Side Channel 18 & $12 / 16 / 2013$ & 5 & 35 \\
Arrah Wanna Side Channel & $12 / 16 / 2013$ & 1 & 7 \\
Still Creek & $01 / 15 / 2013$ & 3 & 21 \\
TOTALS & & 33 & 231 \\
\hline
\end{tabular}




\section{Methods}

Water samples were collected by Burke Strobel, Portland Water Bureau, using sterile syringes, and $15 \mathrm{~mL}$ of the sample were added to a $50 \mathrm{~mL}$ Falcon ${ }^{\circledR}$ tube containing $1.5 \mathrm{~mL}$ sodium acetate and 33 $\mathrm{mL} 200$-proof EtOH. Falcon ${ }^{\circledR}$ tubes were chilled in a cooler in the field and placed in a refrigerator within 6 hours of sample collection. Samples were shipped to the U.S. Geological Survey (USGS) Snake River Field Station on January 30, 2014. Samples were sealed in plastic bags and surrounded with ice. Samples arrived in good condition-cool, no leakage. Samples were stored at $-20{ }^{\circ} \mathrm{C}$ in a refrigerator until DNA extraction from March 24 to June 11, 2014.

DNA was extracted from samples using methods described in Ficetola and others (2008). Samples were first centrifuged at 5,500 g for 35 minutes at $6{ }^{\circ} \mathrm{C}$. The supernatant was discarded. The remaining pellet was then subjected to DNA extraction using a Qiagen DNeasy ${ }^{\circledR}$ Blood \& Tissue Kit (Qiagen $\mathrm{GmbH}$, Hilden, Germany), following the manufacturers protocol, with the only exception being that the final elution was done using $100 \mu \mathrm{L} \mathrm{AE} \mathrm{buffer} \mathrm{to} \mathrm{further} \mathrm{concentrate} \mathrm{DNA.} \mathrm{To} \mathrm{reduce} \mathrm{the}$ effects of inhibitors that may be present in stream water, all samples were purified by post-extraction spin-column purification using OneStep ${ }^{\mathrm{TM}}$ PCR Inhibitor Removal Kit (Zymo Research, Irvine, California; Mckee and others, 2015). Purified DNA was stored in $0.5 \mathrm{~mL}$ cryo-vials at $-20{ }^{\circ} \mathrm{C}$.

We used an existing Chinook molecular assay (Laramie and others, 2015) for analysis of Chinook eDNA. The Coho assay was designed by Marshal Hoy (USGS). Molecular probes for both species were labeled with 6FAM at the 5 ' end and a minor groove binding non-fluorescent quencher (MGB-NFQ; Integrated DNA Technologies, Inc., Coralville, Iowa) at the 3' end. Additional assay specifications are shown in table 2. Both assays were screened for specificity using DNA extracted from non-target tissue samples. None of the non-target DNA, in concentrations likely to be encountered in a natural system, was amplified using the Chinook and Coho molecular assays. DNA from tissue samples used to determine the specificity of the qPCR assays were extracted using a Qiagen DNeasy Blood \& Tissue Kit (Qiagen, GmbH, Hilden, Germany), following the manufacturer's protocol. Extracted DNA was stored in $0.5 \mathrm{~mL}$ cryo-vials at $-20{ }^{\circ} \mathrm{C}$.

Table 2. Specifications of molecular assays for Coho salmon (O. kisutch) and Chinook salmon (0. tshawytscha).

\begin{tabular}{|c|c|c|c|c|c|}
\hline Target species & Region & Base pairs & F-primer & R-primer & Probe \\
\hline $\begin{array}{l}{ }^{1} \text { O. kisutch } \\
\text { (Coho) }\end{array}$ & ${ }^{3} \mathrm{CYTB}$ & 114 & $\begin{array}{l}\text { CCT TGG TGG } \\
\text { CGG ATA TAC } \\
\text { TTA TCT TA }\end{array}$ & $\begin{array}{l}\text { GAA CTAG GAA } \\
\text { GAT GGC GAA } \\
\text { GTA GAT C }\end{array}$ & $\begin{array}{l}\text { 6FAM-TGG AAC } \\
\text { ACC CAT TCA } \\
\text { T-MGBNFQ }\end{array}$ \\
\hline $\begin{array}{l}{ }^{2} O . t \text { shawytscha } \\
\text { (Chinook) }\end{array}$ & ${ }^{4} \mathrm{COI}$ & 90 & $\begin{array}{l}\text { CTG GCA CMG } \\
\text { GGT GAA CAG } \\
\text { TCT ACC }\end{array}$ & $\begin{array}{l}\text { AAT GAA GGG } \\
\text { AGA AGA TCG } \\
\text { TYA GAT CA }\end{array}$ & $\begin{array}{l}\text { 6FAM-CTC CTG } \\
\text { CGT GGG CTA G } \\
\text { MBGNFQ }\end{array}$ \\
\hline
\end{tabular}

${ }^{1}$ Molecular assay developed by Marshal Hoy, Research Scientist, U.S. Geological Survey.

${ }^{2}$ Molecular assay developed by Laramie and others (2015).

${ }^{3} \mathrm{CYTB}$, cytochrome $\mathrm{b}$ region of the mitochondrial genome.

${ }^{4} \mathrm{COI}$, cytochrome oxidase subunit I region of the mitochondrial genome. 
Reactions for qPCR analysis consisted of $15 \mu \mathrm{L}$ total volume, consisting of $0.06 \mu \mathrm{L}$ F-primer, $0.06 \mu \mathrm{L}$ R-primer, $0.03 \mu \mathrm{L}$ probe, $0.6 \mu \mathrm{L}$ AE buffer, $3.75 \mu \mathrm{L}$ DNase-free $\mathrm{H}_{2} \mathrm{O}, 7.5 \mu \mathrm{L}$ Quantitect Multiplex MasterMix, and $3 \mu \mathrm{L}$ sample DNA extract. All qPCR analysis was conducted on a Life Technologies 7300 Real-time qPCR System (Thermo Fisher Scientific, Inc., Waltham, Massachusetts). All qPCR reactions were singleplex.

Reactions using the Chinook assay used thermo-cycler conditions as follows: 2 minute warm up at $50{ }^{\circ} \mathrm{C}, 15$ minute initial heat activation at $95^{\circ} \mathrm{C}$, followed by 50 cycles of 15 seconds denaturation at $94{ }^{\circ} \mathrm{C}$ and 60 seconds annealing/extension at $70^{\circ} \mathrm{C}$. Data were collected during the annealing/extension stage.

Reactions using the Coho assay utilized thermo-cycler conditions as follows: 2 minute warm up at $50{ }^{\circ} \mathrm{C}, 15$-minute initial heat activation at $95^{\circ} \mathrm{C}$, followed by 50 cycles of 15 seconds denaturation at $94{ }^{\circ} \mathrm{C}$ and 60 seconds annealing/extension at $60^{\circ} \mathrm{C}$. Data were collected during the annealing/extension stage.

\section{Molecular Assay Performance}

Standard curves for both Coho and Chinook salmon were created using eight-fold serial dilutions of DNA extracted from fin tissues of their respective species (fig. 2a, 2b).

(a)

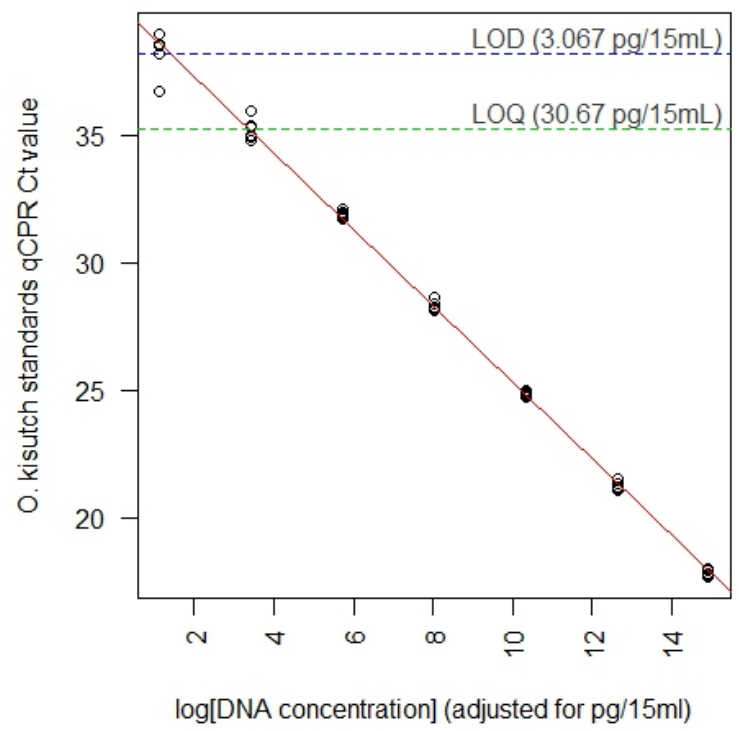

(b)

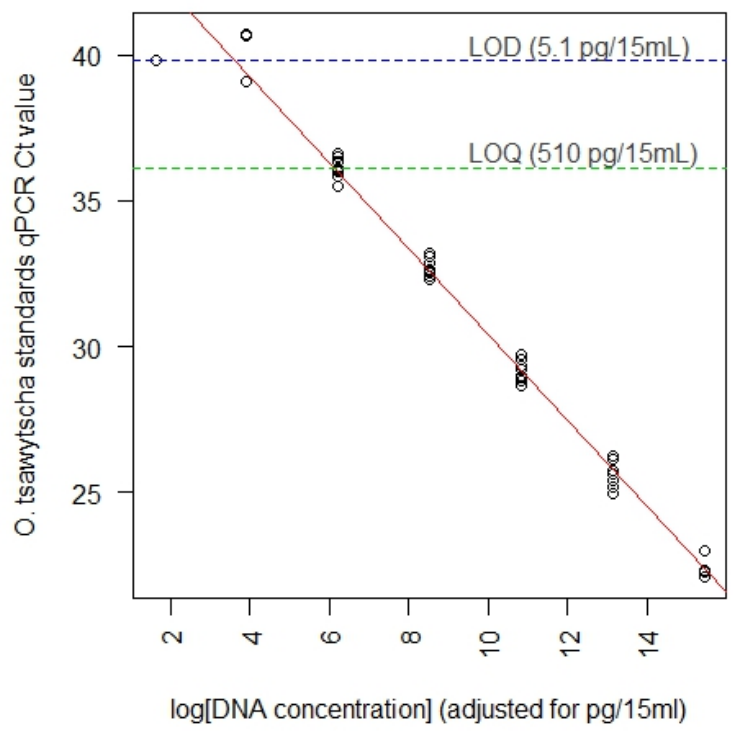

Figure 2. Graphs showing (a) Coho salmon (0. kisutch) DNA Standards (10-fold dilutions), Slope: -3.452282, Intercept: $23.026682, \mathrm{R}^{2}: 0.997334$, Limit of Detection (LOD), based on standard dilutions: $3.067 \times 10^{-5} \mathrm{ng} / \mu \mathrm{L}(3.067$ $\mathrm{pg} / 15 \mathrm{~mL}$, mean $\mathrm{Ct}=38.18962$ ), Limit of Quantitation (LOQ), based on standard dilutions: $3.067 \mathrm{e}-004 \mathrm{ng} / \mu \mathrm{L} \mathrm{L}$ (30.67 pg/15 mL, mean Ct $=35.26478$ ) and (b) Chinook salmon (0. tshawytscha) DNA Standards (10-fold dilutions), Slope: -3.394368 , Intercept: $28.193533, \mathrm{R}^{2}$ : 0.984555, LOD, based on standard dilutions: $5.1 \mathrm{e}-005 \mathrm{ng} / \mathrm{\mu L}$ $(5.1 \mathrm{pg} / 15 \mathrm{~mL}$, mean $\mathrm{Ct}=39.7955), \mathrm{LOQ}$, based on standard dilutions: $0.0051 \mathrm{ng} / \mu \mathrm{L}(510 \mathrm{pg} / 15 \mathrm{~mL}$, mean Ct = 36.12991). 


\section{Data Analysis}

An analysis of variance (ANOVA) was performed to compare mean eDNA concentrations (Coho and Chinook salmon) of each sample group; gravel, water, redds of both species, and three unknown redds. Tukey's Honestly Significant Difference test (multiple comparisons of means, 95percent family-wise confidence level) was then performed to determine which group means were significantly different from each other. Means comparisons were plotted, along with 95-percent familywise confidence intervals. All statistical analyses were performed using R Statistical software (R Core Team, 2013).

\section{Preliminary Results}

Analysis of negative controls.-None of the negative controls amplified using Coho and Chinook salmon molecular assays, including (1) distilled water negative-controls collected at each sampling site, (2) DNA extraction laboratory negative-controls, and (3) qPCR non-template controls. These preliminary findings indicate that no within-site (among samples) or among-site contamination occurred in the field, nor did any contamination interfere with sample analysis.

Sample analysis using the Coho assay.-Analysis conducted using the Coho molecular assay detected eDNA concentrations ranging from 0 to $1,568.88 \mathrm{pg} / 15 \mathrm{~mL}$, with a mean of 106.11 and median of 2.88 (fig. 3a). The sample type "O. kisutch redds" produced the highest mean concentration of Coho eDNA (494.65 pg/15 mL). Means for "O. tshawytscha redds," "gravel," and "water column" were 71.69 $\mathrm{pg} / 15 \mathrm{~mL}, 103.95 \mathrm{pg} / \mathrm{mL}$, and $10.1 \mathrm{pg} / \mathrm{mL}$, respectively. ANOVA indicated a statistical difference between means of the sample group types (Chinook salmon redds, Coho salmon redds, gravel, water; table 3). A Tukey's HSD means comparison indicated only three group mean comparisons were statistically significant; Coho salmon eDNA concentrations for Coho salmon redds differed from gravel, water column, and Chinook salmon redds (table 4). Figure 4a indicates the direction and difference between the various means comparisons performed along with 95 percent family-wise confidence levels. 
Figure 3. Boxplots showing (a) distribution of Coho salmon (O. kistuch) eDNA concentrations at each sample site type, and (b) distribution of Chinook salmon ( $O$. tshawytscha) eDNA concentrations at each sample site type. Thick horizontal lines represent sample medians, boxes represent quartiles, whiskers extend to minimum/maximum (excluding outliers), and circles represent outliers.

Table 3. Analysis of Variance (ANOVA) table for differences in Coho salmon (O. kisutch) eDNA among sample types.

\begin{tabular}{lcccccc}
\hline & $\begin{array}{c}\text { Degrees } \\
\text { of } \\
\text { freedom }\end{array}$ & $\begin{array}{c}\text { Sum of } \\
\text { squares }\end{array}$ & $\begin{array}{c}\text { Mean } \\
\text { Square }\end{array}$ & F value & $\operatorname{Pr}(>\mathrm{F})$ \\
\hline Sample type & 6 & 1847497 & 307916 & 8.1409 & $0.0000019^{* * *}$ & \\
Residuals & 59 & 2231569 & 37823 & & & 1 \\
Significance codes: & $0^{\text {‘**** }}$ & $0.001^{\text {‘** }}$ & $0.01^{* *}$ & 0.05 ' & $0.1^{\prime \prime}$ & 1 \\
\hline
\end{tabular}

Response: Site replicate mean [eDNA] $(\mathrm{pg} / 15 \mathrm{~mL})$

Table 4. Tukey multiple comparisons of means with 95 percent family-wise confidence level for Coho salmon $(0$. kisutch) eDNA among sample types.

\begin{tabular}{lcccc}
\hline \multicolumn{1}{c}{ Sample type } & Difference & $\begin{array}{c}\text { Lower } \\
\text { bound }\end{array}$ & Upper bound & Adjusted p value \\
\hline O. kisutch redd - gravel & 390.692957 & 125.2171 & 656.1688 & 0.0006345 \\
O. tshawytscha redd - O. kisutch redd & -422.953533 & -688.4294 & -157.4777 & 0.0001746 \\
Water - O. kisutch redd & -484.550148 & -698.8329 & -270.2674 & 0.0000001 \\
\hline
\end{tabular}

Fit: $\operatorname{aov}($ formula $=$ Site replicate mean $[e D N A](p g / 15 \mathrm{~mL}) \sim$ Sample type, data $=0$. kisutch eDNA) 
(a)

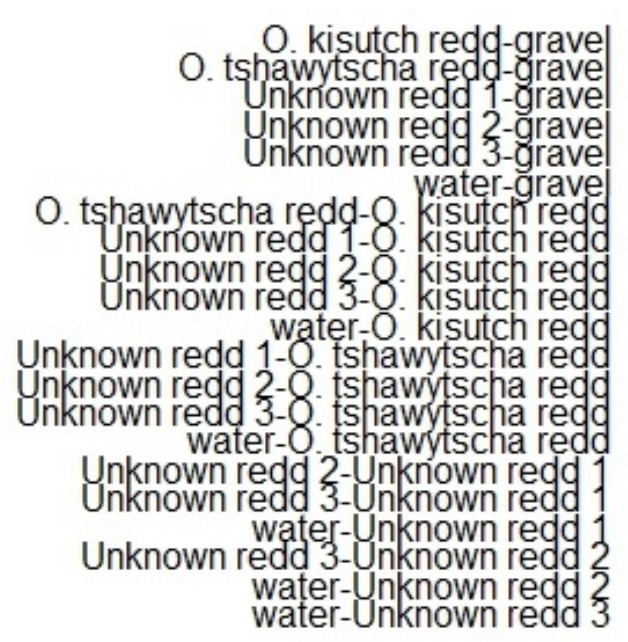

(b)

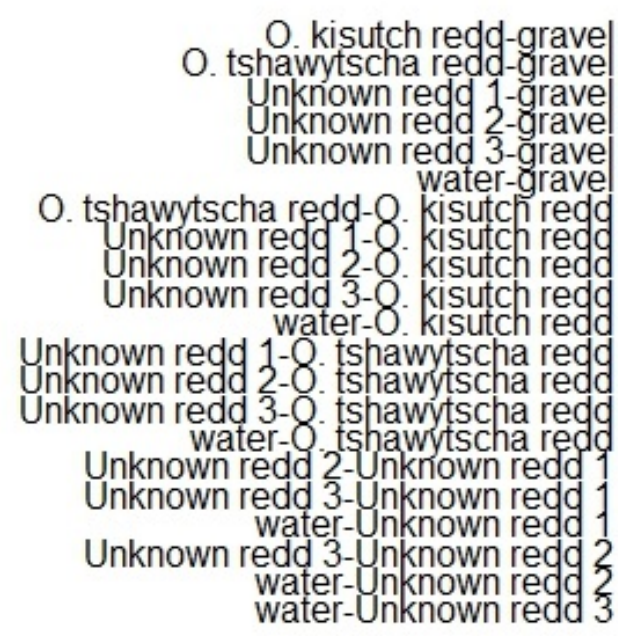

$95 \%$ family-wise confidence level

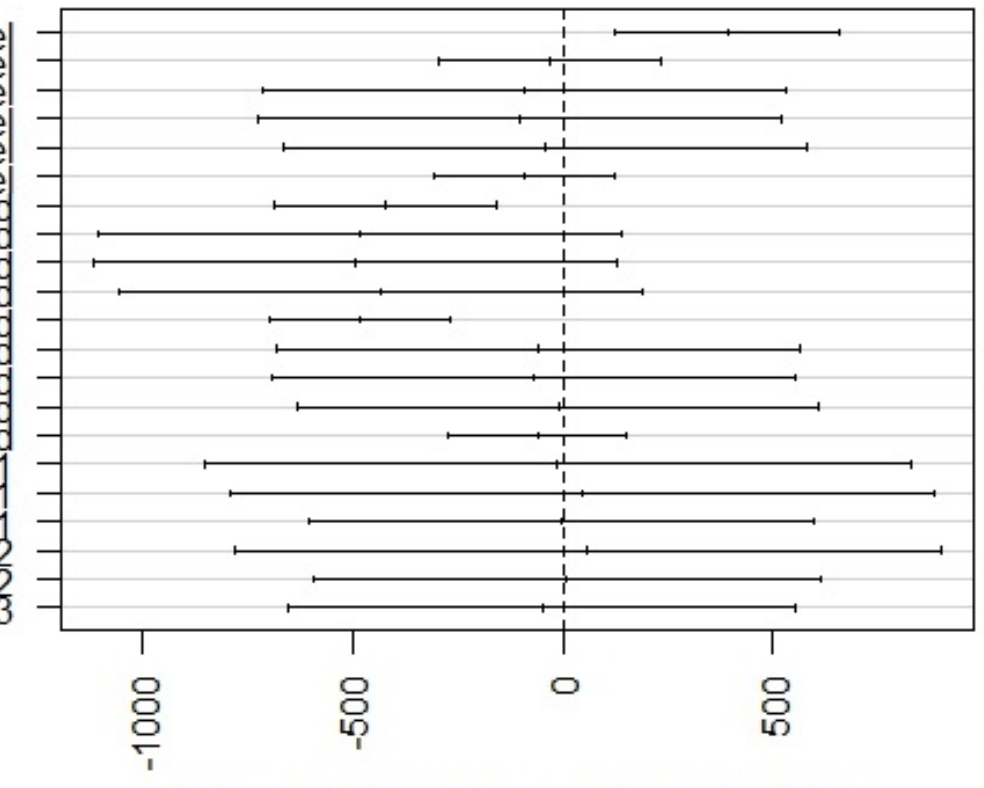

$95 \%$ family-wise confidence level

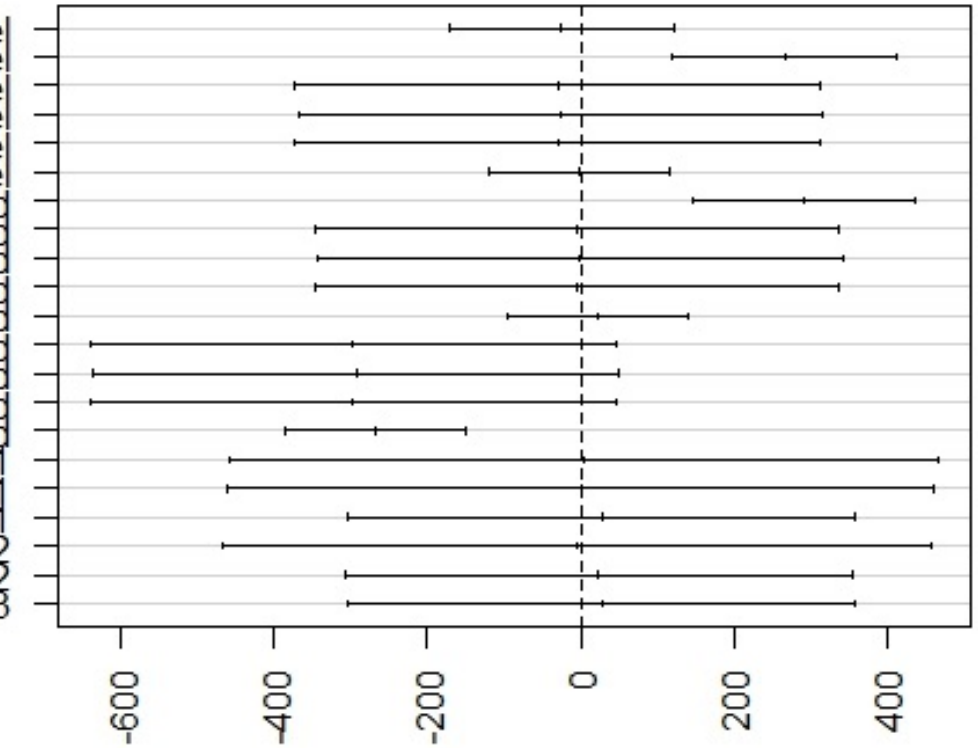

Figure 4. Graphs showing (a) comparisons of mean Coho salmon ( 0 . kisutch) eDNA concentrations between all site types, (b) comparisons of mean Chinook salmon ( 0 . tshawytscha) eDNA concentrations between all site types. $X$-axis represents mean-difference deviation from zero $(\mathrm{pg} / 15 \mathrm{~mL})$. 
Sample analysis using the Chinook assay.-Analysis conducted using the Chinook molecular assay detected eDNA concentrations ranging from 0 to $870.69 \mathrm{pg} / 15 \mathrm{~mL}$, with a mean of 63.98 and median of 4.57 (fig. 3b). The sample type "O. tshawytscha redds" produced the highest concentration of Chinook eDNA (296.64 pg/15 mL). Means for "O. kisutch redds," "gravel," and "water column" were $4.83 \mathrm{pg} / 15 \mathrm{~mL}, 29.89 \mathrm{pg} / \mathrm{mL}$, and $27.43 \mathrm{pg} / \mathrm{mL}$, respectively. ANOVA indicated a statistical difference between means of the sample group types (Chinook salmon redds, Coho salmon redds, gravel, water; table 5). A Tukey's HSD means comparison indicated only three group mean-comparisons were statistically significant; Chinook salmon eDNA concentrations found at Chinook salmon redds differed from gravel, water column, and Coho salmon redds (table 6). Figure 4b indicates the direction and difference between the various means comparisons performed along with 95 percent family-wise confidence intervals.

Table 5. Analysis of Variance (ANOVA) table for differences in Chinook salmon (0. tshawytscha) environmental DNA (eDNA) among sample types.

\begin{tabular}{|c|c|c|c|c|c|}
\hline & $\begin{array}{l}\text { Degrees } \\
\text { of } \\
\text { freedom }\end{array}$ & $\begin{array}{l}\text { Sum of } \\
\text { squares }\end{array}$ & $\begin{array}{l}\text { Mean } \\
\text { Square }\end{array}$ & $F$ value & $\operatorname{Pr}(>F)$ \\
\hline Sample type & 6 & 643758 & 107293 & 9.3578 & $3.318 \mathrm{e}-07 * * *$ \\
\hline Residuals & 59 & 676473 & 11466 & & \\
\hline Significance codes: & $0 ; * * *$, & $0.001 * * *$ & $0.01 * *$ & $0.05^{\prime} ?$ & $0.1^{\prime}$, \\
\hline
\end{tabular}

Response: Site replicate mean [eDNA] (pg/15 mL)

Table 6. Tukey multiple comparisons of means with 95 percent family-wise confidence level for Chinook salmon (0. tshawytscha) environmental DNA (eDNA) among sample types.

\begin{tabular}{lcccc}
\hline \multicolumn{1}{c}{ Sample type } & Difference & Lower bound & Upper bound & Adjusted p-value \\
\hline O. tshawytscha redd - gravel & 266.7449 & 120.5793 & 412.91037 & 0.0000133 \\
O. tshawytsch redd - O. kisutch redd & 291.8099 & 145.6444 & 437.97538 & 0.0000018 \\
Water - O. tshawytscha redd & -269.2079 & -387.1876 & -151.22823 & 0.0000001 \\
\hline
\end{tabular}

Fit: $\operatorname{aov}($ formula $=$ Site replicate mean $[e D N A](p g / 15 \mathrm{~mL}) \sim$ Sample type, data $=$ O. tshawytscha eDNA) 
Sample analysis and assignment of three Unknown redds. - Chinook salmon mean eDNA concentrations at Unknown redd 1, Unknown redd 2, and Unknown redd 3 were $0.00 \pm 0.00 \mathrm{SE}, 4.14 \pm$ 4.14SE, and $0.00 \pm 0.00 \mathrm{SE}$, respectively. Coho salmon mean eDNA concentrations at Unknown redd 1, Unknown redd 2, and Unknown redd 3 were $12.86 \pm 6.32 \mathrm{SE}, 0.72 \pm 0.54 \mathrm{SE}$, and 59.71 $\pm 59.71 \mathrm{SE}$, respectively. The eDNA concentrations (for both species) recovered from these unknown redds was either zero or within the background levels of water or gravel. Therefore, none of the three unknown redds could confidently be assigned to either Chinook or Coho redds. However, Coho salmon eDNA concentrations were higher at Unknown redd 1 and Unknown redd 3, while Chinook salmon eDNA concentrations were only detected at Unknown redd 2.

Note: Two sets of unknown redd sample vials (a set consists of three replicates of redd, three replicates of water, and one DI negative control) were labeled as "Unk 1." It was not possible to determine which sample set was mislabeled, as both sets were otherwise identical. The vials were isolated and shipped in separate plastic bags, a process that allowed for discrimination between the two sample sets despite the identical labels. Therefore, one set of samples was arbitrarily converted in the laboratory to "Unk 2" prior to qPCR analysis. This change did not affect analysis or results.

\section{Acknowledgments}

Coho salmon molecular assay was developed by Marshal Hoy, Research Scientist, U.S. Geological Survey. We thank Boise State University for use of some equipment and laboratory space.

\section{References Cited}

Ficetola, G.F., Miaud, C., Pompanon, F., and Taberlet, P., 2008, Species detection using environmental DNA from water samples: Biology Letters, v. 4, p. 423-425.

Laramie, M.B., Pilliod, D.S., and Goldberg, C.S., 2015, Characterizing the distribution of an endangered salmonid using environmental DNA analysis: Biological Conservation, v. 183, p. 29-37, http://dx.doi.org/10.1016/j.biocon.2014.11.025.

McKee, A.M., Spear, S.F., and Pierson, T.W., 2015, The effect of dilution and the use of a postextraction nucleic acid purification column on the accuracy, precision, and inhibition of environmental DNA samples: Biological Conservation, v. 183, p. 70-76.

R Development Core Team, 2013, R-A language and environment for statistical computing: Vienna, Austria, R Foundation for Statistical Computing, ISBN: 3-900051-07-0. [Also available at http://www.R-project.org/.] 


\section{Appendix 1. Environmental DNA (eDNA) Concentrations for Coho Salmon (0. kisutch) and Chinook salmon (0. tshawytscha) from All Sites Sampled in the Sandy River Basin, northwestern Oregon, fall and winter 2013}

\begin{tabular}{|c|c|c|c|c|c|c|c|}
\hline Sample & Date & Location & Detector & $\begin{array}{c}\text { eDNA } \\
\text { (pg/15mL) }\end{array}$ & Species & Sample type & Replicate \\
\hline CK01RA & $11 / 14 / 2013$ & Bull Run River & O. kisutch CytB & 0.0000 & O. tshawytscha (Chinook) & Redd & 1 \\
\hline CK01RB & $11 / 14 / 2013$ & Bull Run River & O. kisutch CytB & 0.0000 & O. tshawytscha (Chinook) & Redd & 2 \\
\hline CK01RC & $11 / 14 / 2013$ & Bull Run River & O. kisutch CytB & 0.0000 & O. tshawytscha (Chinook) & Redd & 3 \\
\hline CK01WA & $11 / 14 / 2013$ & Bull Run River & O. kisutch CytB & 0.0000 & O. tshawytscha (Chinook) & water & 1 \\
\hline CK01WB & $11 / 14 / 2013$ & Bull Run River & O. kisutch CytB & 0.0000 & O. tshawytscha (Chinook) & water & 2 \\
\hline CK01WC & $11 / 14 / 2013$ & Bull Run River & O. kisutch CytB & 0.0000 & O. tshawytscha (Chinook) & water & 3 \\
\hline CK02RA & $11 / 14 / 2013$ & Bull Run River & O. kisutch CytB & 0.0000 & O. tshawytscha (Chinook) & Redd & 1 \\
\hline CK02RB & $11 / 14 / 2013$ & Bull Run River & O. kisutch CytB & 41.1153 & O. tshawytscha (Chinook) & Redd & 2 \\
\hline CK02RC & $11 / 14 / 2013$ & Bull Run River & O. kisutch CytB & 0.0000 & O. tshawytscha (Chinook) & Redd & 3 \\
\hline CK02WA & $11 / 14 / 2013$ & Bull Run River & O. kisutch CytB & 0.0000 & O. tshawytscha (Chinook) & water & 1 \\
\hline CK02WB & $11 / 14 / 2013$ & Bull Run River & O. kisutch CytB & 0.0000 & O. tshawytscha (Chinook) & water & 2 \\
\hline CK02WC & $11 / 14 / 2013$ & Bull Run River & O. kisutch CytB & 0.0000 & O. tshawytscha (Chinook) & water & 3 \\
\hline CK03RA & $11 / 14 / 2013$ & Bull Run River & O. kisutch CytB & 0.0000 & O. tshawytscha (Chinook) & Redd & 1 \\
\hline CK03RB & $11 / 14 / 2013$ & Bull Run River & O. kisutch CytB & 0.0000 & O. tshawytscha (Chinook) & Redd & 2 \\
\hline CK03RC & $11 / 14 / 2013$ & Bull Run River & O. kisutch CytB & 0.0000 & O. tshawytscha (Chinook) & Redd & 3 \\
\hline CK03WA & $11 / 14 / 2013$ & Bull Run River & O. kisutch CytB & 0.0000 & O. tshawytscha (Chinook) & water & 1 \\
\hline CK03WB & $11 / 14 / 2013$ & Bull Run River & O. kisutch CytB & 0.0000 & O. tshawytscha (Chinook) & water & 2 \\
\hline CK03WC & $11 / 14 / 2013$ & Bull Run River & O. kisutch CytB & 0.0000 & O. tshawytscha (Chinook) & water & 3 \\
\hline CK04RA & $11 / 14 / 2013$ & Bull Run River & O. kisutch CytB & 0.0000 & O. tshawytscha (Chinook) & Redd & 1 \\
\hline CK04RB & $11 / 14 / 2013$ & Bull Run River & O. kisutch CytB & 0.0000 & O. tshawytscha (Chinook) & Redd & 2 \\
\hline CK04RC & $11 / 14 / 2013$ & Bull Run River & O. kisutch CytB & 0.0000 & O. tshawytscha (Chinook) & Redd & 3 \\
\hline CK04WA & $11 / 14 / 2013$ & Bull Run River & O. kisutch CytB & 0.0000 & O. tshawytscha (Chinook) & water & 1 \\
\hline CK04WB & $11 / 14 / 2013$ & Bull Run River & O. kisutch CytB & 0.2080 & O. tshawytscha (Chinook) & water & 2 \\
\hline CK04WC & $11 / 14 / 2013$ & Bull Run River & O. kisutch CytB & 0.0000 & O. tshawytscha (Chinook) & water & 3 \\
\hline
\end{tabular}




\begin{tabular}{|c|c|c|c|c|c|c|c|}
\hline Sample & Date & Location & Detector & $\begin{array}{c}\text { eDNA } \\
\text { (pg/15mL) }\end{array}$ & Species & Sample type & Replicate \\
\hline CK05RA & $11 / 14 / 2013$ & Bull Run River & O. kisutch CytB & 2.1067 & O. tshawytscha (Chinook) & Redd & 1 \\
\hline CK05RB & $11 / 14 / 2013$ & Bull Run River & O. kisutch CytB & 1.7400 & O. tshawytscha (Chinook) & Redd & 2 \\
\hline CK05RC & $11 / 14 / 2013$ & Bull Run River & O. kisutch CytB & 2.1300 & O. tshawytscha (Chinook) & Redd & 3 \\
\hline CK05WA & $11 / 14 / 2013$ & Bull Run River & O. kisutch CytB & 0.1987 & O. tshawytscha (Chinook) & water & 1 \\
\hline CK05WB & $11 / 14 / 2013$ & Bull Run River & O. kisutch CytB & 0.1847 & O. tshawytscha (Chinook) & water & 2 \\
\hline CK05WC & $11 / 14 / 2013$ & Bull Run River & O. kisutch CytB & 0.0000 & O. tshawytscha (Chinook) & water & 3 \\
\hline CK06RA & $11 / 14 / 2013$ & Bull Run River & O. kisutch CytB & 1.6367 & O. tshawytscha (Chinook) & Redd & 1 \\
\hline CK06RB & $11 / 14 / 2013$ & Bull Run River & O. kisutch CytB & 2.2663 & O. tshawytscha (Chinook) & Redd & 2 \\
\hline CK06RC & $11 / 14 / 2013$ & Bull Run River & O. kisutch CytB & 0.4167 & O. tshawytscha (Chinook) & Redd & 3 \\
\hline CK06WA & $11 / 14 / 2013$ & Bull Run River & O. kisutch CytB & 0.0000 & O. tshawytscha (Chinook) & water & 1 \\
\hline CK06WB & $11 / 14 / 2013$ & Bull Run River & O. kisutch CytB & 0.5763 & O. tshawytscha (Chinook) & water & 2 \\
\hline CK06WC & $11 / 14 / 2013$ & Bull Run River & O. kisutch CytB & 1.0133 & O. tshawytscha (Chinook) & water & 3 \\
\hline CK07RA & $11 / 14 / 2013$ & Bull Run River & O. kisutch CytB & 211.6590 & O. tshawytscha (Chinook) & Redd & 1 \\
\hline CK07RB & $11 / 14 / 2013$ & Bull Run River & O. kisutch CytB & 319.2550 & O. tshawytscha (Chinook) & Redd & 2 \\
\hline CK07RC & $11 / 14 / 2013$ & Bull Run River & O. kisutch CytB & 333.0243 & O. tshawytscha (Chinook) & Redd & 3 \\
\hline CK07WA & $11 / 14 / 2013$ & Bull Run River & O. kisutch CytB & 41.7900 & O. tshawytscha (Chinook) & water & 1 \\
\hline CK07WB & $11 / 14 / 2013$ & Bull Run River & O. kisutch CytB & 0.0000 & O. tshawytscha (Chinook) & water & 2 \\
\hline CK07WC & $11 / 14 / 2013$ & Bull Run River & O. kisutch CytB & 29.4000 & O. tshawytscha (Chinook) & water & 3 \\
\hline CK08RA & $11 / 14 / 2013$ & Bull Run River & O. kisutch CytB & 95.8007 & O. tshawytscha (Chinook) & Redd & 1 \\
\hline CK08RB & $11 / 14 / 2013$ & Bull Run River & O. kisutch CytB & 760.4840 & O. tshawytscha (Chinook) & Redd & 2 \\
\hline CK08RC & $11 / 14 / 2013$ & Bull Run River & O. kisutch CytB & 365.7090 & O. tshawytscha (Chinook) & Redd & 3 \\
\hline CK08WA & $11 / 14 / 2013$ & Bull Run River & O. kisutch CytB & 0.0000 & O. tshawytscha (Chinook) & water & 1 \\
\hline CK08WB & $11 / 14 / 2013$ & Bull Run River & O. kisutch CytB & 10.7333 & O. tshawytscha (Chinook) & water & 2 \\
\hline CK08WC & $11 / 14 / 2013$ & Bull Run River & O. kisutch CytB & 0.0000 & O. tshawytscha (Chinook) & water & 3 \\
\hline CK09RA & $11 / 14 / 2013$ & Bull Run River & O. kisutch CytB & 0.7730 & O. tshawytscha (Chinook) & Redd & 1 \\
\hline CK09RB & $11 / 14 / 2013$ & Bull Run River & O. kisutch CytB & 7.2533 & O. tshawytscha (Chinook) & Redd & 2 \\
\hline CK09RC & $11 / 14 / 2013$ & Bull Run River & O. kisutch CytB & 0.6067 & O. tshawytscha (Chinook) & Redd & 3 \\
\hline CK09WA & $11 / 14 / 2013$ & Bull Run River & O. kisutch CytB & 0.0000 & O. tshawytscha (Chinook) & water & 1 \\
\hline CK09WB & $11 / 14 / 2013$ & Bull Run River & O. kisutch CytB & 0.0000 & O. tshawytscha (Chinook) & water & 2 \\
\hline CK09WC & $11 / 14 / 2013$ & Bull Run River & O. kisutch CytB & 0.0000 & O. tshawytscha (Chinook) & water & 3 \\
\hline
\end{tabular}




\begin{tabular}{|c|c|c|c|c|c|c|c|}
\hline Sample & Date & Location & Detector & $\begin{array}{c}\text { eDNA } \\
\text { (pg/15mL) }\end{array}$ & Species & Sample type & Replicate \\
\hline CK10RA & $11 / 14 / 2013$ & Bull Run River & O. kisutch CytB & 0.5240 & O. tshawytscha (Chinook) & Redd & 1 \\
\hline CK10RB & $11 / 14 / 2013$ & Bull Run River & O. kisutch CytB & 1.0600 & O. tshawytscha (Chinook) & Redd & 2 \\
\hline CK10RC & $11 / 14 / 2013$ & Bull Run River & O. kisutch CytB & 3.2233 & O. tshawytscha (Chinook) & Redd & 3 \\
\hline CK10WA & $11 / 14 / 2013$ & Bull Run River & O. kisutch CytB & 0.7700 & O. tshawytscha (Chinook) & water & 1 \\
\hline CK10WB & $11 / 14 / 2013$ & Bull Run River & O. kisutch CytB & 0.0000 & O. tshawytscha (Chinook) & water & 2 \\
\hline CK10WC & $11 / 14 / 2013$ & Bull Run River & O. kisutch CytB & 0.0000 & O. tshawytscha (Chinook) & water & 3 \\
\hline CO01RA & $12 / 18 / 2013$ & Sixes Creek & O. kisutch CytB & 873.1010 & O. kisutch (Coho) & Redd & 1 \\
\hline CO01RB & $12 / 18 / 2013$ & Sixes Creek & O. kisutch CytB & 540.3803 & O. kisutch (Coho) & Redd & 2 \\
\hline $\mathrm{CO} 01 \mathrm{RC}$ & $12 / 18 / 2013$ & Sixes Creek & O. kisutch CytB & 336.4547 & O. kisutch (Coho) & Redd & 3 \\
\hline CO01WA & $12 / 18 / 2013$ & Sixes Creek & O. kisutch CytB & 85.8000 & O. kisutch (Coho) & water & 1 \\
\hline CO01WB & $12 / 18 / 2013$ & Sixes Creek & O. kisutch CytB & 53.1010 & O. kisutch (Coho) & water & 2 \\
\hline CO01WC & $12 / 18 / 2013$ & Sixes Creek & O. kisutch CytB & 0.0000 & O. kisutch (Coho) & water & 3 \\
\hline CO02RA & $12 / 18 / 2013$ & Sixes Creek & O. kisutch CytB & 0.0000 & O. kisutch (Coho) & Redd & 1 \\
\hline $\mathrm{CO} 02 \mathrm{RB}$ & $12 / 18 / 2013$ & Sixes Creek & O. kisutch CytB & 320.9270 & O. kisutch (Coho) & Redd & 2 \\
\hline $\mathrm{CO} 02 \mathrm{RC}$ & $12 / 18 / 2013$ & Sixes Creek & O. kisutch CytB & 0.0000 & O. kisutch (Coho) & Redd & 3 \\
\hline CO02WA & $12 / 18 / 2013$ & Sixes Creek & O. kisutch CytB & 12.5667 & O. kisutch (Coho) & water & 1 \\
\hline $\mathrm{CO} 02 \mathrm{WB}$ & $12 / 18 / 2013$ & Sixes Creek & O. kisutch CytB & 4.2000 & O. kisutch (Coho) & water & 2 \\
\hline $\mathrm{CO} 02 \mathrm{WC}$ & $12 / 18 / 2013$ & Sixes Creek & O. kisutch CytB & 13.4000 & O. kisutch (Coho) & water & 3 \\
\hline CO03RA & $12 / 16 / 2013$ & Side Channel 18 & O. kisutch CytB & 0.0000 & O. kisutch (Coho) & Redd & 1 \\
\hline CO03RB & $12 / 16 / 2013$ & Side Channel 18 & O. kisutch CytB & 0.0000 & O. kisutch (Coho) & Redd & 2 \\
\hline CO03RC & $12 / 16 / 2013$ & Side Channel 18 & O. kisutch CytB & 1022.4900 & O. kisutch (Coho) & Redd & 3 \\
\hline CO03WA & $12 / 16 / 2013$ & Side Channel 18 & O. kisutch CytB & 2.3600 & O. kisutch (Coho) & water & 1 \\
\hline CO03WB & $12 / 16 / 2013$ & Side Channel 18 & O. kisutch CytB & 3.0100 & O. kisutch (Coho) & water & 2 \\
\hline CO03WC & $12 / 16 / 2013$ & Side Channel 18 & O. kisutch CytB & 5.3300 & O. kisutch (Coho) & water & 3 \\
\hline CO04RA & $12 / 18 / 2013$ & Sixes Creek & O. kisutch CytB & 154.4717 & O. kisutch (Coho) & Redd & 1 \\
\hline CO04RB & $12 / 18 / 2013$ & Sixes Creek & O. kisutch CytB & 32.7667 & O. kisutch (Coho) & Redd & 2 \\
\hline CO04RC & $12 / 18 / 2013$ & Sixes Creek & O. kisutch CytB & 10.8267 & O. kisutch (Coho) & Redd & 3 \\
\hline CO04WA & $12 / 18 / 2013$ & Sixes Creek & O. kisutch CytB & 1.2533 & O. kisutch (Coho) & water & 1 \\
\hline CO04WB & $12 / 18 / 2013$ & Sixes Creek & O. kisutch CytB & 3.3267 & O. kisutch (Coho) & water & 2 \\
\hline CO04WC & $12 / 18 / 2013$ & Sixes Creek & O. kisutch CytB & 2.2993 & O. kisutch (Coho) & water & 3 \\
\hline
\end{tabular}




\begin{tabular}{|c|c|c|c|c|c|c|c|}
\hline Sample & Date & Location & Detector & $\begin{array}{c}\text { eDNA } \\
\text { (pg/15mL) }\end{array}$ & Species & Sample type & Replicate \\
\hline CO05RA & $12 / 16 / 2013$ & Side Channel 18 & O. kisutch CytB & 1101.0497 & O. kisutch (Coho) & Redd & 1 \\
\hline CO05RB & $12 / 16 / 2013$ & Side Channel 18 & O. kisutch CytB & 466.4827 & O. kisutch (Coho) & Redd & 2 \\
\hline CO05RC & $12 / 16 / 2013$ & Side Channel 18 & O. kisutch CytB & 581.6717 & O. kisutch (Coho) & Redd & 3 \\
\hline CO05WA & $12 / 16 / 2013$ & Side Channel 18 & O. kisutch CytB & 40.3530 & O. kisutch (Coho) & water & 1 \\
\hline CO05WB & $12 / 16 / 2013$ & Side Channel 18 & O. kisutch CytB & 16.9333 & O. kisutch (Coho) & water & 2 \\
\hline CO05WC & $12 / 16 / 2013$ & Side Channel 18 & O. kisutch CytB & 18.1000 & O. kisutch (Coho) & water & 3 \\
\hline CO06RA & $12 / 16 / 2013$ & Side Channel 18 & O. kisutch CytB & 79.3717 & O. kisutch (Coho) & Redd & 1 \\
\hline CO06RB & $12 / 16 / 2013$ & Side Channel 18 & O. kisutch CytB & 792.9467 & O. kisutch (Coho) & Redd & 2 \\
\hline CO06RC & $12 / 16 / 2013$ & Side Channel 18 & O. kisutch CytB & 365.4017 & O. kisutch (Coho) & Redd & 3 \\
\hline CO06WA & $12 / 16 / 2013$ & Side Channel 18 & O. kisutch CytB & 1.2700 & O. kisutch (Coho) & water & 1 \\
\hline CO06WB & $12 / 16 / 2013$ & Side Channel 18 & O. kisutch CytB & 0.7017 & O. kisutch (Coho) & water & 2 \\
\hline CO06WC & $12 / 16 / 2013$ & Side Channel 18 & O. kisutch CytB & 2.1533 & O. kisutch (Coho) & water & 3 \\
\hline CO07RA & $12 / 16 / 2013$ & $\begin{array}{l}\text { Arrah Wanna } \\
\text { Side Channel }\end{array}$ & O. kisutch CytB & 538.9067 & O. kisutch (Coho) & Redd & 1 \\
\hline CO07RB & $12 / 16 / 2013$ & $\begin{array}{l}\text { Arrah Wanna } \\
\text { Side Channel }\end{array}$ & O. kisutch CytB & 358.3360 & O. kisutch (Coho) & Redd & 2 \\
\hline CO07RC & $12 / 16 / 2013$ & $\begin{array}{l}\text { Arrah Wanna } \\
\text { Side Channel }\end{array}$ & O. kisutch CytB & 183.6263 & O. kisutch (Coho) & Redd & 3 \\
\hline CO07WA & $12 / 16 / 2013$ & $\begin{array}{l}\text { Arrah Wanna } \\
\text { Side Channel }\end{array}$ & O. kisutch CytB & 5.0467 & O. kisutch (Coho) & water & 1 \\
\hline $\mathrm{CO} 07 \mathrm{WB}$ & $12 / 16 / 2013$ & $\begin{array}{l}\text { Arrah Wanna } \\
\text { Side Channel }\end{array}$ & O. kisutch CytB & 5.1600 & O. kisutch (Coho) & water & 2 \\
\hline $\mathrm{CO} 07 \mathrm{WC}$ & $12 / 16 / 2013$ & $\begin{array}{l}\text { Arrah Wanna } \\
\text { Side Channel }\end{array}$ & O. kisutch CytB & 2.2967 & O. kisutch (Coho) & water & 3 \\
\hline CO08RA & $12 / 16 / 2013$ & Side Channel 18 & O. kisutch CytB & 1989.5000 & O. kisutch (Coho) & Redd & 1 \\
\hline CO08RB & $12 / 16 / 2013$ & Side Channel 18 & O. kisutch CytB & 70.2000 & O. kisutch (Coho) & Redd & 2 \\
\hline CO08RC & $12 / 16 / 2013$ & Side Channel 18 & O. kisutch CytB & 3.7667 & O. kisutch (Coho) & Redd & 3 \\
\hline CO08WA & $12 / 16 / 2013$ & Side Channel 18 & O. kisutch CytB & 1.2567 & O. kisutch (Coho) & water & 1 \\
\hline CO08WB & $12 / 16 / 2013$ & Side Channel 18 & O. kisutch CytB & 1.2467 & O. kisutch (Coho) & water & 2 \\
\hline CO08WC & $12 / 16 / 2013$ & Side Channel 18 & O. kisutch CytB & 0.0000 & O. kisutch (Coho) & water & 3 \\
\hline CO09RA & $12 / 16 / 2013$ & Side Channel 18 & O. kisutch CytB & 223.5923 & O. kisutch (Coho) & Redd & 1 \\
\hline CO09RB & $12 / 16 / 2013$ & Side Channel 18 & O. kisutch CytB & 22.4000 & O. kisutch (Coho) & Redd & 2 \\
\hline CO09RC & $12 / 16 / 2013$ & Side Channel 18 & O. kisutch CytB & 64.0667 & O. kisutch (Coho) & Redd & 3 \\
\hline CO09WA & $12 / 16 / 2013$ & Side Channel 18 & O. kisutch CytB & 1.4370 & O. kisutch (Coho) & water & 1 \\
\hline
\end{tabular}




\begin{tabular}{|c|c|c|c|c|c|c|c|}
\hline Sample & Date & Location & Detector & $\begin{array}{c}\text { eDNA } \\
\text { (pg/15mL) }\end{array}$ & Species & Sample type & Replicate \\
\hline CO09WB & $12 / 16 / 2013$ & Side Channel 18 & O. kisutch CytB & 2.3200 & O. kisutch (Coho) & water & 2 \\
\hline CO09WC & $12 / 16 / 2013$ & Side Channel 18 & O. kisutch CytB & 2.5967 & O. kisutch (Coho) & water & 3 \\
\hline CO10RA & $12 / 18 / 2013$ & Sixes Creek & O. kisutch CytB & 32.3667 & O. kisutch (Coho) & Redd & 1 \\
\hline CO10RB & $12 / 18 / 2013$ & Sixes Creek & O. kisutch CytB & 8.8033 & O. kisutch (Coho) & Redd & 2 \\
\hline CO10RC & $12 / 18 / 2013$ & Sixes Creek & O. kisutch CytB & 4665.4833 & O. kisutch (Coho) & Redd & 3 \\
\hline CO10WA & $12 / 18 / 2013$ & Sixes Creek & O. kisutch CytB & 1.2310 & O. kisutch (Coho) & water & 1 \\
\hline CO10WB & $12 / 18 / 2013$ & Sixes Creek & O. kisutch CytB & 0.2007 & O. kisutch (Coho) & water & 2 \\
\hline CO10WC & $12 / 18 / 2013$ & Sixes Creek & O. kisutch CytB & 0.1843 & O. kisutch (Coho) & water & 3 \\
\hline GR01A & $11 / 26 / 2013$ & Bull Run River & O. kisutch CytB & 0.7667 & None & Gravel & 1 \\
\hline GR01B & $11 / 26 / 2013$ & Bull Run River & O. kisutch CytB & 0.0000 & None & Gravel & 2 \\
\hline GR01C & $11 / 26 / 2013$ & Bull Run River & O. kisutch CytB & 4.9167 & None & Gravel & 3 \\
\hline GR01WA & $11 / 26 / 2013$ & Bull Run River & O. kisutch CytB & 0.0000 & None & water & 1 \\
\hline GR01WB & $11 / 26 / 2013$ & Bull Run River & O. kisutch CytB & 0.0000 & None & water & 2 \\
\hline GR01WC & $11 / 26 / 2013$ & Bull Run River & O. kisutch CytB & 0.0000 & None & water & 3 \\
\hline GR02A & $11 / 26 / 2013$ & Bull Run River & O. kisutch CytB & 0.0000 & None & Gravel & 1 \\
\hline GR02B & $11 / 26 / 2013$ & Bull Run River & O. kisutch CytB & 0.0000 & None & Gravel & 2 \\
\hline GR02C & $11 / 26 / 2013$ & Bull Run River & O. kisutch CytB & 110.5487 & None & Gravel & 3 \\
\hline GR02WA & $11 / 26 / 2013$ & Bull Run River & O. kisutch CytB & 0.0000 & None & water & 1 \\
\hline GR02WB & $11 / 26 / 2013$ & Bull Run River & O. kisutch CytB & 0.0000 & None & water & 2 \\
\hline GR02WC & $11 / 26 / 2013$ & Bull Run River & O. kisutch CytB & 0.0000 & None & water & 3 \\
\hline GR03A & $11 / 26 / 2013$ & Bull Run River & O. kisutch CytB & 0.0000 & None & Gravel & 1 \\
\hline GR03B & $11 / 26 / 2013$ & Bull Run River & O. kisutch CytB & 0.7647 & None & Gravel & 2 \\
\hline GR03C & $11 / 26 / 2013$ & Bull Run River & O. kisutch CytB & 0.4500 & None & Gravel & 3 \\
\hline GR03WA & $11 / 26 / 2013$ & Bull Run River & O. kisutch CytB & 0.0000 & None & water & 1 \\
\hline GR03WB & $11 / 26 / 2013$ & Bull Run River & O. kisutch CytB & 0.0000 & None & water & 2 \\
\hline GR03WC & $11 / 26 / 2013$ & Bull Run River & O. kisutch CytB & 0.0000 & None & water & 3 \\
\hline GR04A & $11 / 26 / 2013$ & Bull Run River & O. kisutch CytB & 38.3617 & None & Gravel & 1 \\
\hline GR04B & $11 / 26 / 2013$ & Bull Run River & O. kisutch CytB & 102.1077 & None & Gravel & 2 \\
\hline GR04C & $11 / 26 / 2013$ & Bull Run River & O. kisutch CytB & 0.0000 & None & Gravel & 3 \\
\hline GR04WA & $11 / 26 / 2013$ & Bull Run River & O. kisutch CytB & 0.0000 & None & water & 1 \\
\hline
\end{tabular}




\begin{tabular}{|c|c|c|c|c|c|c|c|}
\hline Sample & Date & Location & Detector & $\begin{array}{c}\text { eDNA } \\
\text { (pg/15mL) }\end{array}$ & Species & Sample type & Replicate \\
\hline GR04WB & $11 / 26 / 2013$ & Bull Run River & O. kisutch CytB & 0.0000 & None & water & 2 \\
\hline GR04WC & $11 / 26 / 2013$ & Bull Run River & O. kisutch CytB & 0.0000 & None & water & 3 \\
\hline GR05A & $11 / 26 / 2013$ & Bull Run River & O. kisutch CytB & 288.8107 & None & Gravel & 1 \\
\hline GR05B & $11 / 26 / 2013$ & Bull Run River & O. kisutch CytB & 254.0207 & None & Gravel & 2 \\
\hline GR05C & $11 / 26 / 2013$ & Bull Run River & O. kisutch CytB & 175.3717 & None & Gravel & 3 \\
\hline GR05WA & $11 / 26 / 2013$ & Bull Run River & O. kisutch CytB & 87.3133 & None & water & 1 \\
\hline GR05WB & $11 / 26 / 2013$ & Bull Run River & O. kisutch CytB & 0.0000 & None & water & 2 \\
\hline GR05WC & $11 / 26 / 2013$ & Bull Run River & O. kisutch CytB & 0.0000 & None & water & 3 \\
\hline GR06A & $11 / 26 / 2013$ & Bull Run River & O. kisutch CytB & 183.3780 & None & Gravel & 1 \\
\hline GR06B & $11 / 26 / 2013$ & Bull Run River & O. kisutch CytB & 113.1340 & None & Gravel & 2 \\
\hline GR06C & $11 / 26 / 2013$ & Bull Run River & O. kisutch CytB & 1151.8350 & None & Gravel & 3 \\
\hline GR06WA & $11 / 26 / 2013$ & Bull Run River & O. kisutch CytB & 0.0000 & None & water & 1 \\
\hline GR06WB & $11 / 26 / 2013$ & Bull Run River & O. kisutch CytB & 0.0000 & None & water & 2 \\
\hline GR06WC & $11 / 26 / 2013$ & Bull Run River & O. kisutch CytB & 0.0000 & None & water & 3 \\
\hline GR07A & $11 / 26 / 2013$ & Bull Run River & O. kisutch CytB & 0.6267 & None & Gravel & 1 \\
\hline GR07B & $11 / 26 / 2013$ & Bull Run River & O. kisutch CytB & 0.0076 & None & Gravel & 2 \\
\hline GR07C & $11 / 26 / 2013$ & Bull Run River & O. kisutch CytB & 9.7167 & None & Gravel & 3 \\
\hline GR07WA & $11 / 26 / 2013$ & Bull Run River & O. kisutch CytB & 0.0000 & None & water & 1 \\
\hline GR07WB & $11 / 26 / 2013$ & Bull Run River & O. kisutch CytB & 0.0000 & None & water & 2 \\
\hline GR07WC & $11 / 26 / 2013$ & Bull Run River & O. kisutch CytB & 0.4267 & None & water & 3 \\
\hline GR08A & $11 / 26 / 2013$ & Bull Run River & O. kisutch CytB & 0.0000 & None & Gravel & 1 \\
\hline GR08B & $11 / 26 / 2013$ & Bull Run River & O. kisutch CytB & 0.0000 & None & Gravel & 2 \\
\hline GR08C & $11 / 26 / 2013$ & Bull Run River & O. kisutch CytB & 0.0000 & None & Gravel & 3 \\
\hline GR08WA & $11 / 26 / 2013$ & Bull Run River & O. kisutch CytB & 0.0000 & None & water & 1 \\
\hline GR08WB & $11 / 26 / 2013$ & Bull Run River & O. kisutch CytB & 0.0000 & None & water & 2 \\
\hline GR08WC & $11 / 26 / 2013$ & Bull Run River & O. kisutch CytB & 0.0000 & None & water & 3 \\
\hline GR09A & $11 / 26 / 2013$ & Bull Run River & O. kisutch CytB & 81.7003 & None & Gravel & 1 \\
\hline GR09B & $11 / 26 / 2013$ & Bull Run River & O. kisutch CytB & 0.0000 & None & Gravel & 2 \\
\hline GR09C & $11 / 26 / 2013$ & Bull Run River & O. kisutch CytB & 583.7227 & None & Gravel & 3 \\
\hline GR09WA & $11 / 26 / 2013$ & Bull Run River & O. kisutch CytB & 0.0000 & None & water & 1 \\
\hline
\end{tabular}




\begin{tabular}{|c|c|c|c|c|c|c|c|}
\hline Sample & Date & Location & Detector & $\begin{array}{c}\text { eDNA } \\
\text { (pg/15mL) }\end{array}$ & Species & Sample type & Replicate \\
\hline GR09WB & $11 / 26 / 2013$ & Bull Run River & O. kisutch CytB & 203.8467 & None & water & 2 \\
\hline GR09WC & $11 / 26 / 2013$ & Bull Run River & O. kisutch CytB & 0.0000 & None & water & 3 \\
\hline GR10A & $11 / 26 / 2013$ & Bull Run River & O. kisutch CytB & 16.7333 & None & Gravel & 1 \\
\hline GR10B & $11 / 26 / 2013$ & Bull Run River & O. kisutch CytB & 0.0080 & None & Gravel & 2 \\
\hline GR10C & $11 / 26 / 2013$ & Bull Run River & O. kisutch CytB & 1.6200 & None & Gravel & 3 \\
\hline GR10WA & $11 / 26 / 2013$ & Bull Run River & O. kisutch CytB & 0.0000 & None & water & 1 \\
\hline GR10WB & $11 / 26 / 2013$ & Bull Run River & O. kisutch CytB & 0.2557 & None & water & 2 \\
\hline GR10WC & $11 / 26 / 2013$ & Bull Run River & O. kisutch CytB & 0.0000 & None & water & 3 \\
\hline UNK01RA & $1 / 15 / 2013$ & Still Creek & O. kisutch CytB & 3.8813 & Unknown & Redd & 1 \\
\hline UNK01RB & $1 / 15 / 2013$ & Still Creek & O. kisutch CytB & 25.0667 & Unknown & Redd & 2 \\
\hline UNK01RC & $1 / 15 / 2013$ & Still Creek & O. kisutch CytB & 9.6367 & Unknown & Redd & 3 \\
\hline UNK01WA & $1 / 15 / 2013$ & Still Creek & O. kisutch CytB & 0.0000 & Unknown & water & 1 \\
\hline UNK01WB & $1 / 15 / 2013$ & Still Creek & O. kisutch CytB & 0.0000 & Unknown & water & 2 \\
\hline UNK01WC & $1 / 15 / 2013$ & Still Creek & O. kisutch CytB & 0.0000 & Unknown & water & 3 \\
\hline UNK02RA & $1 / 15 / 2013$ & Still Creek & O. kisutch CytB & 1.7833 & Unknown & Redd & 1 \\
\hline UNK02RB & $1 / 15 / 2013$ & Still Creek & O. kisutch CytB & 0.3733 & Unknown & Redd & 2 \\
\hline UNK02RC & $1 / 15 / 2013$ & Still Creek & O. kisutch CytB & 0.0000 & Unknown & Redd & 3 \\
\hline UNK02WA & $1 / 15 / 2013$ & Still Creek & O. kisutch CytB & 0.0000 & Unknown & water & 1 \\
\hline UNK02WB & $1 / 15 / 2013$ & Still Creek & O. kisutch CytB & 0.0000 & Unknown & water & 2 \\
\hline UNK02WC & $1 / 15 / 2013$ & Still Creek & O. kisutch CytB & 284.8343 & Unknown & water & 3 \\
\hline UNK03RA & $1 / 15 / 2013$ & Still Creek & O. kisutch CytB & 179.1353 & Unknown & Redd & 1 \\
\hline UNK03RB & $1 / 15 / 2013$ & Still Creek & O. kisutch CytB & 0.0000 & Unknown & Redd & 2 \\
\hline UNK03RC & $1 / 15 / 2013$ & Still Creek & O. kisutch CytB & 0.0000 & Unknown & Redd & 3 \\
\hline UNK03WA & $1 / 15 / 2013$ & Still Creek & O. kisutch CytB & 0.0000 & Unknown & water & 1 \\
\hline UNK03WB & $1 / 15 / 2013$ & Still Creek & O. kisutch CytB & 0.0000 & Unknown & water & 2 \\
\hline UNK03WC & $1 / 15 / 2013$ & Still Creek & O. kisutch CytB & 48.8367 & Unknown & water & 3 \\
\hline CK01RA & $11 / 14 / 2013$ & Bull Run River & O. tshawytscha COI & 178.5853 & O. tshawytscha (Chinook) & Redd & 1 \\
\hline CK01RB & $11 / 14 / 2013$ & Bull Run River & O. tshawytscha COI & 260.4847 & O. tshawytscha (Chinook) & Redd & 2 \\
\hline CK01RC & $11 / 14 / 2013$ & Bull Run River & O. tshawytscha COI & 89.0983 & O. tshawytscha (Chinook) & Redd & 3 \\
\hline CK01WA & $11 / 14 / 2013$ & Bull Run River & O. tshawytscha COI & 87.6147 & O. tshawytscha (Chinook) & water & 1 \\
\hline
\end{tabular}




\begin{tabular}{|c|c|c|c|c|c|c|c|}
\hline Sample & Date & Location & Detector & $\begin{array}{c}\text { eDNA } \\
\text { (pg/15mL) }\end{array}$ & Species & Sample type & Replicate \\
\hline CK01WB & $11 / 14 / 2013$ & Bull Run River & O. tshawytscha COI & 218.5640 & O. tshawytscha (Chinook) & water & 2 \\
\hline CK01WC & $11 / 14 / 2013$ & Bull Run River & O. tshawytscha COI & 16.1000 & O. tshawytscha (Chinook) & water & 3 \\
\hline CK02RA & $11 / 14 / 2013$ & Bull Run River & O. tshawytscha COI & 261.5873 & O. tshawytscha (Chinook) & Redd & 1 \\
\hline CK02RB & $11 / 14 / 2013$ & Bull Run River & O. tshawytscha COI & 166.3707 & O. tshawytscha (Chinook) & Redd & 2 \\
\hline CK02RC & $11 / 14 / 2013$ & Bull Run River & O. tshawytscha COI & 219.1917 & O. tshawytscha (Chinook) & Redd & 3 \\
\hline CK02WA & $11 / 14 / 2013$ & Bull Run River & O. tshawytscha COI & 78.9000 & O. tshawytscha (Chinook) & water & 1 \\
\hline CK02WB & $11 / 14 / 2013$ & Bull Run River & O. tshawytscha COI & 199.3390 & O. tshawytscha (Chinook) & water & 2 \\
\hline CK02WC & $11 / 14 / 2013$ & Bull Run River & O. tshawytscha COI & 378.6940 & O. tshawytscha (Chinook) & water & 3 \\
\hline CK03RA & $11 / 14 / 2013$ & Bull Run River & O. tshawytscha COI & 344.0073 & O. tshawytscha (Chinook) & Redd & 1 \\
\hline CK03RB & $11 / 14 / 2013$ & Bull Run River & O. tshawytscha COI & 335.5297 & O. tshawytscha (Chinook) & Redd & 2 \\
\hline CK03RC & $11 / 14 / 2013$ & Bull Run River & O. tshawytscha COI & 87.0577 & O. tshawytscha (Chinook) & Redd & 3 \\
\hline CK03WA & $11 / 14 / 2013$ & Bull Run River & O. tshawytscha COI & 13.7333 & O. tshawytscha (Chinook) & water & 1 \\
\hline CK03WB & $11 / 14 / 2013$ & Bull Run River & O. tshawytscha COI & 57.9100 & O. tshawytscha (Chinook) & water & 2 \\
\hline CK03WC & $11 / 14 / 2013$ & Bull Run River & O. tshawytscha COI & 35.1333 & O. tshawytscha (Chinook) & water & 3 \\
\hline CK04RA & $11 / 14 / 2013$ & Bull Run River & O. tshawytscha COI & 201.5137 & O. tshawytscha (Chinook) & Redd & 1 \\
\hline CK04RB & $11 / 14 / 2013$ & Bull Run River & O. tshawytscha COI & 259.8697 & O. tshawytscha (Chinook) & Redd & 2 \\
\hline CK04RC & $11 / 14 / 2013$ & Bull Run River & O. tshawytscha COI & 240.3683 & O. tshawytscha (Chinook) & Redd & 3 \\
\hline CK04WA & $11 / 14 / 2013$ & Bull Run River & O. tshawytscha COI & 121.2920 & O. tshawytscha (Chinook) & water & 1 \\
\hline CK04WB & $11 / 14 / 2013$ & Bull Run River & O. tshawytscha COI & 102.2447 & O. tshawytscha (Chinook) & water & 2 \\
\hline CK04WC & $11 / 14 / 2013$ & Bull Run River & O. tshawytscha COI & 47.1667 & O. tshawytscha (Chinook) & water & 3 \\
\hline CK05RA & $11 / 14 / 2013$ & Bull Run River & O. tshawytscha COI & 286.9430 & O. tshawytscha (Chinook) & Redd & 1 \\
\hline CK05RB & $11 / 14 / 2013$ & Bull Run River & O. tshawytscha COI & 63.1770 & O. tshawytscha (Chinook) & Redd & 2 \\
\hline CK05RC & $11 / 14 / 2013$ & Bull Run River & O. tshawytscha COI & 151.5153 & O. tshawytscha (Chinook) & Redd & 3 \\
\hline CK05WA & $11 / 14 / 2013$ & Bull Run River & O. tshawytscha COI & 30.2000 & O. tshawytscha (Chinook) & water & 1 \\
\hline CK05WB & $11 / 14 / 2013$ & Bull Run River & O. tshawytscha COI & 63.3197 & O. tshawytscha (Chinook) & water & 2 \\
\hline CK05WC & $11 / 14 / 2013$ & Bull Run River & O. tshawytscha COI & 20.4000 & O. tshawytscha (Chinook) & water & 3 \\
\hline CK06RA & $11 / 14 / 2013$ & Bull Run River & O. tshawytscha COI & 133.7667 & O. tshawytscha (Chinook) & Redd & 1 \\
\hline CK06RB & $11 / 14 / 2013$ & Bull Run River & O. tshawytscha COI & 280.2207 & O. tshawytscha (Chinook) & Redd & 2 \\
\hline CK06RC & $11 / 14 / 2013$ & Bull Run River & O. tshawytscha COI & 129.2073 & O. tshawytscha (Chinook) & Redd & 3 \\
\hline CK06WA & $11 / 14 / 2013$ & Bull Run River & O. tshawytscha COI & 90.7270 & O. tshawytscha (Chinook) & water & 1 \\
\hline
\end{tabular}




\begin{tabular}{|c|c|c|c|c|c|c|c|}
\hline Sample & Date & Location & Detector & $\begin{array}{c}\text { eDNA } \\
\text { (pg/15mL) }\end{array}$ & Species & Sample type & Replicate \\
\hline CK06WB & $11 / 14 / 2013$ & Bull Run River & O. tshawytscha COI & 124.0723 & O. tshawytscha (Chinook) & water & 2 \\
\hline CK06WC & $11 / 14 / 2013$ & Bull Run River & O. tshawytscha COI & 113.9230 & O. tshawytscha (Chinook) & water & 3 \\
\hline CK07RA & $11 / 14 / 2013$ & Bull Run River & O. tshawytscha COI & 205.3013 & O. tshawytscha (Chinook) & Redd & 1 \\
\hline CK07RB & $11 / 14 / 2013$ & Bull Run River & O. tshawytscha COI & 0.0540 & O. tshawytscha (Chinook) & Redd & 2 \\
\hline CK07RC & $11 / 14 / 2013$ & Bull Run River & O. tshawytscha COI & 113.5967 & O. tshawytscha (Chinook) & Redd & 3 \\
\hline CK07WA & $11 / 14 / 2013$ & Bull Run River & O. tshawytscha COI & 27.9000 & O. tshawytscha (Chinook) & water & 1 \\
\hline CK07WB & $11 / 14 / 2013$ & Bull Run River & O. tshawytscha COI & 34.6667 & O. tshawytscha (Chinook) & water & 2 \\
\hline CK07WC & $11 / 14 / 2013$ & Bull Run River & O. tshawytscha COI & 116.7387 & O. tshawytscha (Chinook) & water & 3 \\
\hline CK08RA & $11 / 14 / 2013$ & Bull Run River & O. tshawytscha COI & 33.0369 & O. tshawytscha (Chinook) & Redd & 1 \\
\hline CK08RB & $11 / 14 / 2013$ & Bull Run River & O. tshawytscha COI & 109.1894 & O. tshawytscha (Chinook) & Redd & 2 \\
\hline CK08RC & $11 / 14 / 2013$ & Bull Run River & O. tshawytscha COI & 1818.2493 & O. tshawytscha (Chinook) & Redd & 3 \\
\hline CK08WA & $11 / 14 / 2013$ & Bull Run River & O. tshawytscha COI & 28.5985 & O. tshawytscha (Chinook) & water & 1 \\
\hline CK08WB & $11 / 14 / 2013$ & Bull Run River & O. tshawytscha COI & 36.2548 & O. tshawytscha (Chinook) & water & 2 \\
\hline CK08WC & $11 / 14 / 2013$ & Bull Run River & O. tshawytscha COI & 7.1027 & O. tshawytscha (Chinook) & water & 3 \\
\hline CK09RA & $11 / 14 / 2013$ & Bull Run River & O. tshawytscha COI & 806.5780 & O. tshawytscha (Chinook) & Redd & 1 \\
\hline CK09RB & $11 / 14 / 2013$ & Bull Run River & O. tshawytscha COI & 1310.4433 & O. tshawytscha (Chinook) & Redd & 2 \\
\hline CK09RC & $11 / 14 / 2013$ & Bull Run River & O. tshawytscha COI & 495.0417 & O. tshawytscha (Chinook) & Redd & 3 \\
\hline CK09WA & $11 / 14 / 2013$ & Bull Run River & O. tshawytscha COI & 53.3333 & O. tshawytscha (Chinook) & water & 1 \\
\hline CK09WB & $11 / 14 / 2013$ & Bull Run River & O. tshawytscha COI & 57.5633 & O. tshawytscha (Chinook) & water & 2 \\
\hline CK09WC & $11 / 14 / 2013$ & Bull Run River & O. tshawytscha COI & 45.1467 & O. tshawytscha (Chinook) & water & 3 \\
\hline CK10RA & $11 / 14 / 2013$ & Bull Run River & O. tshawytscha COI & 89.3580 & O. tshawytscha (Chinook) & Redd & 1 \\
\hline CK10RB & $11 / 14 / 2013$ & Bull Run River & O. tshawytscha COI & 152.3263 & O. tshawytscha (Chinook) & Redd & 2 \\
\hline CK10RC & $11 / 14 / 2013$ & Bull Run River & O. tshawytscha COI & 77.4720 & O. tshawytscha (Chinook) & Redd & 3 \\
\hline CK10WA & $11 / 14 / 2013$ & Bull Run River & O. tshawytscha COI & 76.1620 & O. tshawytscha (Chinook) & water & 1 \\
\hline CK10WB & $11 / 14 / 2013$ & Bull Run River & O. tshawytscha COI & 44.0000 & O. tshawytscha (Chinook) & water & 2 \\
\hline CK10WC & $11 / 14 / 2013$ & Bull Run River & O. tshawytscha COI & 20.1667 & O. tshawytscha (Chinook) & water & 3 \\
\hline CO01RA & $12 / 18 / 2013$ & Sixes Creek & O. tshawytscha COI & 3.9233 & O. kisutch (Coho) & Redd & 1 \\
\hline $\mathrm{CO} 01 \mathrm{RB}$ & $12 / 18 / 2013$ & Sixes Creek & O. tshawytscha COI & 2.5767 & O. kisutch (Coho) & Redd & 2 \\
\hline CO01RC & $12 / 18 / 2013$ & Sixes Creek & O. tshawytscha COI & 0.0000 & O. kisutch (Coho) & Redd & 3 \\
\hline CO01WA & $12 / 18 / 2013$ & Sixes Creek & O. tshawytscha COI & 0.0000 & O. kisutch (Coho) & water & 1 \\
\hline
\end{tabular}




\begin{tabular}{|c|c|c|c|c|c|c|c|}
\hline Sample & Date & Location & Detector & $\begin{array}{c}\text { eDNA } \\
\text { (pg/15mL) }\end{array}$ & Species & Sample type & Replicate \\
\hline CO01WB & $12 / 18 / 2013$ & Sixes Creek & O. tshawytscha COI & 0.0000 & O. kisutch (Coho) & water & 2 \\
\hline CO01WC & $12 / 18 / 2013$ & Sixes Creek & O. tshawytscha COI & 0.0000 & O. kisutch (Coho) & water & 3 \\
\hline CO02RA & $12 / 18 / 2013$ & Sixes Creek & O. tshawytscha COI & 0.0000 & O. kisutch (Coho) & Redd & 1 \\
\hline $\mathrm{CO} 02 \mathrm{RB}$ & $12 / 18 / 2013$ & Sixes Creek & O. tshawytscha COI & 0.0000 & O. kisutch (Coho) & Redd & 2 \\
\hline $\mathrm{CO} 02 \mathrm{RC}$ & $12 / 18 / 2013$ & Sixes Creek & O. tshawytscha COI & 0.0000 & O. kisutch (Coho) & Redd & 3 \\
\hline CO02WA & $12 / 18 / 2013$ & Sixes Creek & O. tshawytscha COI & 0.0000 & O. kisutch (Coho) & water & 1 \\
\hline CO02WB & $12 / 18 / 2013$ & Sixes Creek & O. tshawytscha COI & 0.0000 & O. kisutch (Coho) & water & 2 \\
\hline $\mathrm{CO} 02 \mathrm{WC}$ & $12 / 18 / 2013$ & Sixes Creek & O. tshawytscha COI & 12.1667 & O. kisutch (Coho) & water & 3 \\
\hline CO03RA & $12 / 16 / 2013$ & Side Channel 18 & O. tshawytscha COI & 0.0000 & O. kisutch (Coho) & Redd & 1 \\
\hline CO03RB & $12 / 16 / 2013$ & Side Channel 18 & O. tshawytscha COI & 0.0000 & O. kisutch (Coho) & Redd & 2 \\
\hline CO03RC & $12 / 16 / 2013$ & Side Channel 18 & O. tshawytscha COI & 0.0000 & O. kisutch (Coho) & Redd & 3 \\
\hline CO03WA & $12 / 16 / 2013$ & Side Channel 18 & O. tshawytscha COI & 0.0000 & O. kisutch (Coho) & water & 1 \\
\hline CO03WB & $12 / 16 / 2013$ & Side Channel 18 & O. tshawytscha COI & 0.0000 & O. kisutch (Coho) & water & 2 \\
\hline CO03WC & $12 / 16 / 2013$ & Side Channel 18 & O. tshawytscha COI & 0.0000 & O. kisutch (Coho) & water & 3 \\
\hline CO04RA & $12 / 18 / 2013$ & Sixes Creek & O. tshawytscha COI & 55.3100 & O. kisutch (Coho) & Redd & 1 \\
\hline CO04RB & $12 / 18 / 2013$ & Sixes Creek & O. tshawytscha COI & 78.6197 & O. kisutch (Coho) & Redd & 2 \\
\hline CO04RC & $12 / 18 / 2013$ & Sixes Creek & O. tshawytscha COI & 0.0000 & O. kisutch (Coho) & Redd & 3 \\
\hline CO04WA & $12 / 18 / 2013$ & Sixes Creek & O. tshawytscha COI & 21.4333 & O. kisutch (Coho) & water & 1 \\
\hline CO04WB & $12 / 18 / 2013$ & Sixes Creek & O. tshawytscha COI & 5.9333 & O. kisutch (Coho) & water & 2 \\
\hline CO04WC & $12 / 18 / 2013$ & Sixes Creek & O. tshawytscha COI & 0.0000 & O. kisutch (Coho) & water & 3 \\
\hline CO05RA & $12 / 16 / 2013$ & Side Channel 18 & O. tshawytscha COI & 0.0000 & O. kisutch (Coho) & Redd & 1 \\
\hline CO05RB & $12 / 16 / 2013$ & Side Channel 18 & O. tshawytscha COI & 1.6957 & O. kisutch (Coho) & Redd & 2 \\
\hline CO05RC & $12 / 16 / 2013$ & Side Channel 18 & O. tshawytscha COI & 2.7200 & O. kisutch (Coho) & Redd & 3 \\
\hline CO05WA & $12 / 16 / 2013$ & Side Channel 18 & O. tshawytscha COI & 0.0000 & O. kisutch (Coho) & water & 1 \\
\hline CO05WB & $12 / 16 / 2013$ & Side Channel 18 & O. tshawytscha COI & 2.5333 & O. kisutch (Coho) & water & 2 \\
\hline CO05WC & $12 / 16 / 2013$ & Side Channel 18 & O. tshawytscha COI & 0.0000 & O. kisutch (Coho) & water & 3 \\
\hline CO06RA & $12 / 16 / 2013$ & Side Channel 18 & O. tshawytscha COI & 0.0000 & O. kisutch (Coho) & Redd & 1 \\
\hline CO06RB & $12 / 16 / 2013$ & Side Channel 18 & O. tshawytscha COI & 0.0000 & O. kisutch (Coho) & Redd & 2 \\
\hline CO06RC & $12 / 16 / 2013$ & Side Channel 18 & O. tshawytscha COI & 0.0000 & O. kisutch (Coho) & Redd & 3 \\
\hline CO06WA & $12 / 16 / 2013$ & Side Channel 18 & O. tshawytscha COI & 0.0000 & O. kisutch (Coho) & water & 1 \\
\hline
\end{tabular}




\begin{tabular}{|c|c|c|c|c|c|c|c|}
\hline Sample & Date & Location & Detector & $\begin{array}{c}\text { eDNA } \\
\text { (pg/15mL) }\end{array}$ & Species & Sample type & Replicate \\
\hline CO06WB & $12 / 16 / 2013$ & Side Channel 18 & O. tshawytscha COI & 0.0000 & O. kisutch (Coho) & water & 2 \\
\hline CO06WC & $12 / 16 / 2013$ & Side Channel 18 & O. tshawytscha COI & 0.0000 & O. kisutch (Coho) & water & 3 \\
\hline CO07RA & $12 / 16 / 2013$ & $\begin{array}{l}\text { Arrah Wanna } \\
\text { Side Channel }\end{array}$ & O. tshawytscha COI & 0.0000 & O. kisutch (Coho) & Redd & 1 \\
\hline CO07RB & $12 / 16 / 2013$ & $\begin{array}{l}\text { Arrah Wanna } \\
\text { Side Channel }\end{array}$ & O. tshawytscha COI & 0.0000 & O. kisutch (Coho) & Redd & 2 \\
\hline $\mathrm{CO} 07 \mathrm{RC}$ & $12 / 16 / 2013$ & $\begin{array}{l}\text { Arrah Wanna } \\
\text { Side Channel }\end{array}$ & O. tshawytscha COI & 0.0000 & O. kisutch (Coho) & Redd & 3 \\
\hline CO07WA & $12 / 16 / 2013$ & $\begin{array}{l}\text { Arrah Wanna } \\
\text { Side Channel }\end{array}$ & O. tshawytscha COI & 0.0000 & O. kisutch (Coho) & water & 1 \\
\hline CO07WB & $12 / 16 / 2013$ & $\begin{array}{l}\text { Arrah Wanna } \\
\text { Side Channel }\end{array}$ & O. tshawytscha COI & 0.0000 & O. kisutch (Coho) & water & 2 \\
\hline CO07WC & $12 / 16 / 2013$ & $\begin{array}{l}\text { Arrah Wanna } \\
\text { Side Channel }\end{array}$ & O. tshawytscha COI & 0.0000 & O. kisutch (Coho) & water & 3 \\
\hline CO08RA & $12 / 16 / 2013$ & Side Channel 18 & O. tshawytscha COI & 0.0000 & O. kisutch (Coho) & Redd & 1 \\
\hline CO08RB & $12 / 16 / 2013$ & Side Channel 18 & O. tshawytscha COI & 0.0000 & O. kisutch (Coho) & Redd & 2 \\
\hline CO08RC & $12 / 16 / 2013$ & Side Channel 18 & O. tshawytscha COI & 0.0000 & O. kisutch (Coho) & Redd & 3 \\
\hline CO08WA & $12 / 16 / 2013$ & Side Channel 18 & O. tshawytscha COI & 0.0000 & O. kisutch (Coho) & water & 1 \\
\hline CO08WB & $12 / 16 / 2013$ & Side Channel 18 & O. tshawytscha COI & 0.0000 & O. kisutch (Coho) & water & 2 \\
\hline CO08WC & $12 / 16 / 2013$ & Side Channel 18 & O. tshawytscha COI & 0.0000 & O. kisutch (Coho) & water & 3 \\
\hline CO09RA & $12 / 16 / 2013$ & Side Channel 18 & O. tshawytscha COI & 0.0000 & O. kisutch (Coho) & Redd & 1 \\
\hline CO09RB & $12 / 16 / 2013$ & Side Channel 18 & O. tshawytscha COI & 0.0000 & O. kisutch (Coho) & Redd & 2 \\
\hline CO09RC & $12 / 16 / 2013$ & Side Channel 18 & O. tshawytscha COI & 0.0000 & O. kisutch (Coho) & Redd & 3 \\
\hline CO09WA & $12 / 16 / 2013$ & Side Channel 18 & O. tshawytscha COI & 0.0000 & O. kisutch (Coho) & water & 1 \\
\hline CO09WB & $12 / 16 / 2013$ & Side Channel 18 & O. tshawytscha COI & 0.0000 & O. kisutch (Coho) & water & 2 \\
\hline CO09WC & $12 / 16 / 2013$ & Side Channel 18 & O. tshawytscha COI & 0.0000 & O. kisutch (Coho) & water & 3 \\
\hline CO10RA & $12 / 18 / 2013$ & Sixes Creek & O. tshawytscha COI & 0.0000 & O. kisutch (Coho) & Redd & 1 \\
\hline CO10RB & $12 / 18 / 2013$ & Sixes Creek & O. tshawytscha COI & 0.0000 & O. kisutch (Coho) & Redd & 2 \\
\hline CO10RC & $12 / 18 / 2013$ & Sixes Creek & O. tshawytscha COI & 0.0000 & O. kisutch (Coho) & Redd & 3 \\
\hline CO10WA & $12 / 18 / 2013$ & Sixes Creek & O. tshawytscha COI & 14.9667 & O. kisutch (Coho) & water & 1 \\
\hline CO10WB & $12 / 18 / 2013$ & Sixes Creek & O. tshawytscha COI & 0.0000 & O. kisutch (Coho) & water & 2 \\
\hline CO10WC & $12 / 18 / 2013$ & Sixes Creek & O. tshawytscha COI & 0.0000 & O. kisutch (Coho) & water & 3 \\
\hline GR01A & $11 / 26 / 2013$ & Bull Run River & O. tshawytscha COI & 0.0000 & None & Gravel & 1 \\
\hline \multirow[t]{2}{*}{ GR01B } & $11 / 26 / 2013$ & Bull Run River & O. tshawytscha COI & 0.0000 & None & Gravel & 2 \\
\hline & & & & 20 & & & \\
\hline
\end{tabular}




\begin{tabular}{|c|c|c|c|c|c|c|c|}
\hline Sample & Date & Location & Detector & $\begin{array}{c}\text { eDNA } \\
\text { (pg/15mL) }\end{array}$ & Species & Sample type & Replicate \\
\hline GR01C & $11 / 26 / 2013$ & Bull Run River & O. tshawytscha COI & 36.0667 & None & Gravel & 3 \\
\hline GR01WA & $11 / 26 / 2013$ & Bull Run River & O. tshawytscha COI & 0.0000 & None & water & 1 \\
\hline GR01WB & $11 / 26 / 2013$ & Bull Run River & O. tshawytscha COI & 0.0000 & None & water & 2 \\
\hline GR01WC & $11 / 26 / 2013$ & Bull Run River & O. tshawytscha COI & 0.0000 & None & water & 3 \\
\hline GR02A & $11 / 26 / 2013$ & Bull Run River & O. tshawytscha COI & 6.3667 & None & Gravel & 1 \\
\hline GR02B & $11 / 26 / 2013$ & Bull Run River & O. tshawytscha COI & 0.0000 & None & Gravel & 2 \\
\hline GR02C & $11 / 26 / 2013$ & Bull Run River & O. tshawytscha COI & 0.0000 & None & Gravel & 3 \\
\hline GR02WA & $11 / 26 / 2013$ & Bull Run River & O. tshawytscha COI & 0.0000 & None & water & 1 \\
\hline GR02WB & $11 / 26 / 2013$ & Bull Run River & O. tshawytscha COI & 0.0000 & None & water & 2 \\
\hline GR02WC & $11 / 26 / 2013$ & Bull Run River & O. tshawytscha COI & 0.0000 & None & water & 3 \\
\hline GR03A & $11 / 26 / 2013$ & Bull Run River & O. tshawytscha COI & 239.5760 & None & Gravel & 1 \\
\hline GR03B & $11 / 26 / 2013$ & Bull Run River & O. tshawytscha COI & 168.0500 & None & Gravel & 2 \\
\hline GR03C & $11 / 26 / 2013$ & Bull Run River & O. tshawytscha COI & 6.7333 & None & Gravel & 3 \\
\hline GR03WA & $11 / 26 / 2013$ & Bull Run River & O. tshawytscha COI & 23.7667 & None & water & 1 \\
\hline GR03WB & $11 / 26 / 2013$ & Bull Run River & O. tshawytscha COI & 0.0000 & None & water & 2 \\
\hline GR03WC & $11 / 26 / 2013$ & Bull Run River & O. tshawytscha COI & 0.0000 & None & water & 3 \\
\hline GR04A & $11 / 26 / 2013$ & Bull Run River & O. tshawytscha COI & 0.0000 & None & Gravel & 1 \\
\hline GR04B & $11 / 26 / 2013$ & Bull Run River & O. tshawytscha COI & 0.7200 & None & Gravel & 2 \\
\hline GR04C & $11 / 26 / 2013$ & Bull Run River & O. tshawytscha COI & 1.0067 & None & Gravel & 3 \\
\hline GR04WA & $11 / 26 / 2013$ & Bull Run River & O. tshawytscha COI & 0.0000 & None & water & 1 \\
\hline GR04WB & $11 / 26 / 2013$ & Bull Run River & O. tshawytscha COI & 0.0000 & None & water & 2 \\
\hline GR04WC & $11 / 26 / 2013$ & Bull Run River & O. tshawytscha COI & 0.0000 & None & water & 3 \\
\hline GR05A & $11 / 26 / 2013$ & Bull Run River & O. tshawytscha COI & 0.0000 & None & Gravel & 1 \\
\hline GR05B & $11 / 26 / 2013$ & Bull Run River & O. tshawytscha COI & 0.2835 & None & Gravel & 2 \\
\hline GR05C & $11 / 26 / 2013$ & Bull Run River & O. tshawytscha COI & 0.0000 & None & Gravel & 3 \\
\hline GR05WA & $11 / 26 / 2013$ & Bull Run River & O. tshawytscha COI & 0.0000 & None & water & 1 \\
\hline GR05WB & $11 / 26 / 2013$ & Bull Run River & O. tshawytscha COI & 0.0000 & None & water & 2 \\
\hline GR05WC & $11 / 26 / 2013$ & Bull Run River & O. tshawytscha COI & 0.0000 & None & water & 3 \\
\hline GR06A & $11 / 26 / 2013$ & Bull Run River & O. tshawytscha COI & 53.1492 & None & Gravel & 1 \\
\hline GR06B & $11 / 26 / 2013$ & Bull Run River & O. tshawytscha COI & 21.5795 & None & Gravel & 2 \\
\hline
\end{tabular}




\begin{tabular}{|c|c|c|c|c|c|c|c|}
\hline Sample & Date & Location & Detector & $\begin{array}{c}\text { eDNA } \\
\text { (pg/15mL) }\end{array}$ & Species & Sample type & Replicate \\
\hline GR06C & $11 / 26 / 2013$ & Bull Run River & O. tshawytscha COI & 28.2565 & None & Gravel & 3 \\
\hline GR06WA & $11 / 26 / 2013$ & Bull Run River & O. tshawytscha COI & 50.9030 & None & water & 1 \\
\hline GR06WB & $11 / 26 / 2013$ & Bull Run River & O. tshawytscha COI & 42.5484 & None & water & 2 \\
\hline GR06WC & $11 / 26 / 2013$ & Bull Run River & O. tshawytscha COI & 0.2319 & None & water & 3 \\
\hline GR07A & $11 / 26 / 2013$ & Bull Run River & O. tshawytscha COI & 53.2333 & None & Gravel & 1 \\
\hline GR07B & $11 / 26 / 2013$ & Bull Run River & O. tshawytscha COI & 8.9333 & None & Gravel & 2 \\
\hline GR07C & $11 / 26 / 2013$ & Bull Run River & O. tshawytscha COI & 17.9000 & None & Gravel & 3 \\
\hline GR07WA & $11 / 26 / 2013$ & Bull Run River & O. tshawytscha COI & 0.0000 & None & water & 1 \\
\hline GR07WB & $11 / 26 / 2013$ & Bull Run River & O. tshawytscha COI & 81.7817 & None & water & 2 \\
\hline GR07WC & $11 / 26 / 2013$ & Bull Run River & O. tshawytscha COI & 20.8000 & None & water & 3 \\
\hline GR08A & $11 / 26 / 2013$ & Bull Run River & O. tshawytscha COI & 0.0000 & None & Gravel & 1 \\
\hline GR08B & $11 / 26 / 2013$ & Bull Run River & O. tshawytscha COI & 0.0000 & None & Gravel & 2 \\
\hline GR08C & $11 / 26 / 2013$ & Bull Run River & O. tshawytscha COI & 0.0000 & None & Gravel & 3 \\
\hline GR08WA & $11 / 26 / 2013$ & Bull Run River & O. tshawytscha COI & 0.0000 & None & water & 1 \\
\hline GR08WB & $11 / 26 / 2013$ & Bull Run River & O. tshawytscha COI & 0.0000 & None & water & 2 \\
\hline GR08WC & $11 / 26 / 2013$ & Bull Run River & O. tshawytscha COI & 0.0000 & None & water & 3 \\
\hline GR09A & $11 / 26 / 2013$ & Bull Run River & O. tshawytscha COI & 3.9660 & None & Gravel & 1 \\
\hline GR09B & $11 / 26 / 2013$ & Bull Run River & O. tshawytscha COI & 24.6396 & None & Gravel & 2 \\
\hline GR09C & $11 / 26 / 2013$ & Bull Run River & O. tshawytscha COI & 220.4252 & None & Gravel & 3 \\
\hline GR09WA & $11 / 26 / 2013$ & Bull Run River & O. tshawytscha COI & 0.0000 & None & water & 1 \\
\hline GR09WB & $11 / 26 / 2013$ & Bull Run River & O. tshawytscha COI & 19.3195 & None & water & 2 \\
\hline GR09WC & $11 / 26 / 2013$ & Bull Run River & O. tshawytscha COI & 0.0000 & None & water & 3 \\
\hline GR10A & $11 / 26 / 2013$ & Bull Run River & O. tshawytscha COI & 0.3567 & None & Gravel & 1 \\
\hline GR10B & $11 / 26 / 2013$ & Bull Run River & O. tshawytscha COI & 0.0000 & None & Gravel & 2 \\
\hline GR10C & $11 / 26 / 2013$ & Bull Run River & O. tshawytscha COI & 5.5533 & None & Gravel & 3 \\
\hline GR10WA & $11 / 26 / 2013$ & Bull Run River & O. tshawytscha COI & 0.0000 & None & water & 1 \\
\hline GR10WB & $11 / 26 / 2013$ & Bull Run River & O. tshawytscha COI & 9.8000 & None & water & 2 \\
\hline GR10WC & $11 / 26 / 2013$ & Bull Run River & O. tshawytscha COI & 62.4333 & None & water & 3 \\
\hline UNK01RA & $1 / 15 / 2013$ & Still Creek & O. tshawytscha COI & 0.0000 & Unknown & Redd & 1 \\
\hline UNK01RB & $1 / 15 / 2013$ & Still Creek & O. tshawytscha COI & 0.0000 & Unknown & Redd & 2 \\
\hline
\end{tabular}




\begin{tabular}{|c|c|c|c|c|c|c|c|}
\hline Sample & Date & Location & Detector & $\begin{array}{c}\text { eDNA } \\
\text { (pg/15mL) }\end{array}$ & Species & Sample type & Replicate \\
\hline UNK01RC & $1 / 15 / 2013$ & Still Creek & O. tshawytscha COI & 0.0000 & Unknown & Redd & 3 \\
\hline UNK01WA & $1 / 15 / 2013$ & Still Creek & O. tshawytscha COI & 0.0000 & Unknown & water & 1 \\
\hline UNK01WB & $1 / 15 / 2013$ & Still Creek & O. tshawytscha COI & 0.0000 & Unknown & water & 2 \\
\hline UNK01WC & $1 / 15 / 2013$ & Still Creek & O. tshawytscha COI & 0.0000 & Unknown & water & 3 \\
\hline UNK02RA & $1 / 15 / 2013$ & Still Creek & O. tshawytscha COI & 0.0000 & Unknown & Redd & 1 \\
\hline UNK02RB & $1 / 15 / 2013$ & Still Creek & O. tshawytscha COI & 12.4333 & Unknown & Redd & 2 \\
\hline UNK02RC & $1 / 15 / 2013$ & Still Creek & O. tshawytscha COI & 0.0000 & Unknown & Redd & 3 \\
\hline UNK02WA & $1 / 15 / 2013$ & Still Creek & O. tshawytscha COI & 0.0000 & Unknown & water & 1 \\
\hline UNK02WB & $1 / 15 / 2013$ & Still Creek & O. tshawytscha COI & 0.0000 & Unknown & water & 2 \\
\hline UNK02WC & $1 / 15 / 2013$ & Still Creek & O. tshawytscha COI & 0.0000 & Unknown & water & 3 \\
\hline UNK03RA & $1 / 15 / 2013$ & Still Creek & O. tshawytscha COI & 0.0000 & Unknown & Redd & 1 \\
\hline UNK03RB & $1 / 15 / 2013$ & Still Creek & O. tshawytscha COI & 0.0000 & Unknown & Redd & 2 \\
\hline UNK03RC & $1 / 15 / 2013$ & Still Creek & O. tshawytscha COI & 0.0000 & Unknown & Redd & 3 \\
\hline UNK03WA & $1 / 15 / 2013$ & Still Creek & O. tshawytscha COI & 0.0000 & Unknown & water & 1 \\
\hline UNK03WB & $1 / 15 / 2013$ & Still Creek & O. tshawytscha COI & 0.0000 & Unknown & water & 2 \\
\hline UNK03WC & $1 / 15 / 2013$ & Still Creek & O. tshawytscha COI & 0.0000 & Unknown & water & 3 \\
\hline
\end{tabular}


Appendix 2. Difference $(\Delta)$ Between Mean Environmental DNA (eDNA) Concentrations near the Substrate and in the Water Column (for O. kisutch and O. tshawytscha) at Each Site in the Sandy River Basin, northwestern Oregon, fall and winter 2013

\begin{tabular}{|c|c|c|c|c|c|c|c|c|c|}
\hline Site & $\begin{array}{l}\text { Substrate } \\
\text { eDNA Coho } \\
\text { pg/15mL }\end{array}$ & $\begin{array}{l}\text { Water eDNA } \\
\text { Coho } \\
\text { pg/15mL }\end{array}$ & $\begin{array}{c}\triangle \text { eDNA } \\
\text { Coho }\end{array}$ & $\begin{array}{l}\text { Substrate } \\
\text { eDNA Chinook } \\
\text { pg/15mL }\end{array}$ & $\begin{array}{c}\text { Water eDNA } \\
\text { Chinook } \\
\text { pg/15mL }\end{array}$ & $\begin{array}{l}\triangle \mathrm{eDNA} \\
\text { Chinook }\end{array}$ & Species & Date & Stream \\
\hline $\mathrm{CO} 01$ & 583.3120 & 46.3003 & 537.0117 & 2.1667 & 0.0000 & 2.1667 & Coho & $12 / 18 / 2013$ & Sixes Creek \\
\hline $\mathrm{CO} 02$ & 106.9757 & 10.0556 & 96.9201 & 0.0000 & 4.0556 & -4.0556 & Coho & $12 / 18 / 2013$ & Sixes Creek \\
\hline $\mathrm{CO} 03$ & 340.8300 & 3.5667 & 337.2633 & 0.0000 & 0.0000 & 0.0000 & Coho & $12 / 16 / 2013$ & Side Channel 18 \\
\hline $\mathrm{CO} 04$ & 66.0217 & 2.2931 & 63.7286 & 44.6432 & 9.1222 & 35.5210 & Coho & $12 / 18 / 2013$ & Sixes Creek \\
\hline $\mathrm{CO} 05$ & 716.4013 & 25.1288 & 691.2726 & 1.4719 & 0.8444 & 0.6274 & Coho & $12 / 16 / 2013$ & Side Channel 18 \\
\hline $\mathrm{CO} 06$ & 412.5733 & 1.3750 & 411.1983 & 0.0000 & 0.0000 & 0.0000 & Coho & $12 / 16 / 2013$ & Side Channel 18 \\
\hline $\mathrm{CO} 07$ & 360.2897 & 4.1678 & 356.1219 & 0.0000 & 0.0000 & 0.0000 & Coho & $12 / 16 / 2013$ & $\begin{array}{l}\text { Arrah Wanna Side } \\
\text { Channel }\end{array}$ \\
\hline CO08 & 687.8222 & 0.8344 & 686.9878 & 0.0000 & 0.0000 & 0.0000 & Coho & $12 / 16 / 2013$ & Side Channel 18 \\
\hline $\mathrm{CO} 09$ & 103.3530 & 2.1179 & 101.2351 & 0.0000 & 0.0000 & 0.0000 & Coho & $12 / 16 / 2013$ & Side Channel 18 \\
\hline CO10 & $1,568.8844$ & 0.5387 & $1,568.3458$ & 0.0000 & 4.9889 & -4.9889 & Coho & $12 / 18 / 2013$ & Sixes Creek \\
\hline CK01 & 0.0000 & 0.0000 & 0.0000 & 176.0561 & 107.4262 & 68.6299 & Chinook & $11 / 14 / 2013$ & Bull Run River \\
\hline CK02 & 13.7051 & 0.0000 & 13.7051 & 215.7166 & 218.9777 & -3.2611 & Chinook & $11 / 14 / 2013$ & Bull Run River \\
\hline CK03 & 0.0000 & 0.0000 & 0.0000 & 255.5316 & 35.5922 & 219.9393 & Chinook & $11 / 14 / 2013$ & Bull Run River \\
\hline CK04 & 0.0000 & 0.0693 & -0.0693 & 233.9172 & 90.2344 & 143.6828 & Chinook & $11 / 14 / 2013$ & Bull Run River \\
\hline CK05 & 1.9922 & 0.1278 & 1.8644 & 167.2118 & 37.9732 & 129.2386 & Chinook & $11 / 14 / 2013$ & Bull Run River \\
\hline CK06 & 1.4399 & 0.5299 & 0.9100 & 181.0649 & 109.5741 & 71.4908 & Chinook & $11 / 14 / 2013$ & Bull Run River \\
\hline CK07 & 287.9794 & 23.7300 & 264.2494 & 106.3173 & 59.7684 & 46.5489 & Chinook & $11 / 14 / 2013$ & Bull Run River \\
\hline CK08 & 407.3312 & 3.5778 & 403.7534 & 653.4919 & 23.9853 & 629.5066 & Chinook & $11 / 14 / 2013$ & Bull Run River \\
\hline CK09 & 2.8777 & 0.0000 & 2.8777 & 870.6877 & 52.0144 & 818.6732 & Chinook & $11 / 14 / 2013$ & Bull Run River \\
\hline CK10 & 1.6024 & 0.2567 & 1.3458 & 106.3854 & 46.7762 & 59.6092 & Chinook & $11 / 14 / 2013$ & Bull Run River \\
\hline GR01 & 1.8944 & 0.0000 & 1.8944 & 12.0222 & 0.0000 & 12.0222 & None & $11 / 26 / 2013$ & Bull Run River \\
\hline
\end{tabular}




\begin{tabular}{|c|c|c|c|c|c|c|c|c|c|}
\hline Site & $\begin{array}{c}\text { Substrate } \\
\text { eDNA Coho } \\
\text { pg/15mL }\end{array}$ & $\begin{array}{c}\text { Water eDNA } \\
\text { Coho } \\
\text { pg/15mL }\end{array}$ & $\begin{array}{c}\Delta \text { eDNA } \\
\text { Coho }\end{array}$ & $\begin{array}{c}\text { Substrate } \\
\text { eDNA Chinook } \\
\text { pg/15mL }\end{array}$ & $\begin{array}{c}\text { Water eDNA } \\
\text { Chinook } \\
\text { pg/15mL }\end{array}$ & $\begin{array}{l}\Delta \text { eDNA } \\
\text { Chinook }\end{array}$ & Species & Date & Stream \\
\hline GR02 & 36.8496 & 0.0000 & 36.8496 & 2.1222 & 0.0000 & 2.1222 & None & $11 / 26 / 2013$ & Bull Run River \\
\hline GR03 & 0.4049 & 0.0000 & 0.4049 & 138.1198 & 7.9222 & 130.1976 & None & $11 / 26 / 2013$ & Bull Run River \\
\hline GR04 & 46.8231 & 0.0000 & 46.8231 & 0.5756 & 0.0000 & 0.5756 & None & $11 / 26 / 2013$ & Bull Run River \\
\hline GR05 & 239.4010 & 29.1044 & 210.2966 & 0.0945 & 0.0000 & 0.0945 & None & $11 / 26 / 2013$ & Bull Run River \\
\hline GR06 & 482.7823 & 0.0000 & 482.7823 & 34.3284 & 31.2278 & 3.1006 & None & $11 / 26 / 2013$ & Bull Run River \\
\hline GR07 & 3.4503 & 0.1422 & 3.3081 & 26.6889 & 34.1939 & -7.5050 & None & $11 / 26 / 2013$ & Bull Run River \\
\hline GR08 & 0.0000 & 0.0000 & 0.0000 & 0.0000 & 0.0000 & 0.0000 & None & $11 / 26 / 2013$ & Bull Run River \\
\hline GR09 & 221.8077 & 67.9489 & 153.8588 & 83.0103 & 6.4398 & 76.5704 & None & $11 / 26 / 2013$ & Bull Run River \\
\hline GR10 & 6.1205 & 0.0852 & 6.0352 & 1.9700 & 24.0778 & -22.1078 & None & $11 / 26 / 2013$ & Bull Run River \\
\hline UNK01 & 12.8616 & 0.0000 & 12.8616 & 0.0000 & 0.0000 & 0.0000 & Unknown & $1 / 15 / 2013$ & Still Creek \\
\hline UNK02 & 0.7189 & 94.9448 & -94.2259 & 4.1444 & 0.0000 & 4.1444 & Unknown & $1 / 15 / 2013$ & Still Creek \\
\hline UNK03 & 59.7118 & 16.2789 & 43.4329 & 0.0000 & 0.0000 & 0.0000 & Unknown & $1 / 15 / 2013$ & Still Creek \\
\hline
\end{tabular}



Publishing support provided by the U.S. Geological Survey Science Publishing Network, Tacoma Publishing Service Center

For more information concerning the research in this report, contact the Director, Forest and Rangeland Ecosystem Science Center U.S. Geological Survey

777 NW 9th St., Suite 400

Corvallis, Oregon 97330

http://fresc.usgs.gov/ 


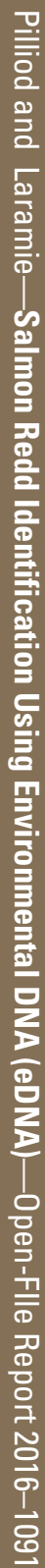

